\title{
Critical Care Medicine
}

\section{Sepsis reduces bone strength before morphological changes are identifiable --Manuscript Draft--}

Manuscript Number:

Full Title:

Article Type:

Keywords:

Corresponding Author:

Corresponding Author's Institution:

First Author:

Order of Authors:

Manuscript Region of Origin:

Abstract:

Response to Reviewers:
CCMED-D-17-00932R2

Sepsis reduces bone strength before morphological changes are identifiable

Online Laboratory Investigation

Functional outcomes; sepsis; bone; fracture

Zudin Puthucheary, MRCP PhD

University College London

London, london UNITED KINGDOM

University College London

Zudin Puthucheary, MRCP PhD

Zudin Puthucheary, MRCP PhD

Yao Sun

Lien Hong Vu

Kaiyang Zeng

ZhiWei Zhang

Ryan Lim

Nicholas Chew

Matthew Cove

\section{SINGAPORE}

Objectives: Survivors of critical illness have an increased incidence of bone fractures. However, early changes in bone strength, and their relationship to structural changes, have not been described. We aimed to characterize early changes in bone functional properties in critical illness and their relationship to changes in bone structure, using a sepsis rodent model.

Design: Experimental Study

Setting: Animal Research Laboratory

Subjects: Adult Sprague-Dawley rats

Interventions: Forty Sprague-Dawley rats were randomised to cecal ligation and puncture (CLP) or sham surgery. Twenty rodents (10 CLP, 10 sham) were sacrificed at 24 hours, and 20 more at 96 hours.

Measurements and main results: Femoral bones were harvested for strength testing, microCT imaging, histological analysis, and multi-frequency scanning probe microscopy (SPM). Fracture loads at the femoral neck were significantly reduced for CLP-exposed rodents at 24 hours $(83.39 \pm 10.1 \mathrm{~N}$ vs. $103.1 \pm 17.6 \mathrm{~N} ; \mathrm{p}=0.014)$ and 96 hours $(81.60 \pm 14.2 \mathrm{~N}$ vs. $95.66 \pm 14.3 \mathrm{~N} ; \mathrm{p}=0.047)$. Using multi-frequency SPM, collagen elastic modulus was lower in CLP-exposed rats at 24 hours $(1.37 \pm 0.2 \mathrm{GPa}$ vs. $6.13 \pm 0.3 \mathrm{GPa} ; \mathrm{p}=0.001)$ and 96 hours $(5.57 \pm 0.5 \mathrm{GPa}$ vs. $6.13 \pm 0.3 \mathrm{GPa} ; \mathrm{p}=0.006)$. Bone mineral elastic modulus was similar at 24 hours, but reduced in CLP-exposed rodents at 96 hours $(75.34 \pm 13.2 \mathrm{GPa}$ vs. $134.4 \pm 8.2 \mathrm{GPa} ; \mathrm{p}<0.001)$. There were no bone architectural or Bone Mineral Density differences by microCT. Similarly, histological analysis demonstrated no difference in collagen and, elastin staining, and Chemokine Receptor type 4, Nuclear Factor Kappa Beta and Tartarate Resistant Acid Phosphatase immunostaining.

Conclusions: In a rodent sepsis model, trabecular bone strength is functionally reduced within 24 hours and is associated with a reduction in collagen and mineral elastic modulus. This is likely to be the result of altered biomechanical properties, rather than increased bone mineral turnover. These data offer both mechanistic insights and may potential guide development of therapeutic interventions.

Reply to Reviewers: Sepsis reduces bone strength 
Reviewer 1: This experimental study adds laboratory based evidence to the emerging literature on increased bone turnover, loss of bone density, and fracture after critical illness. The comparison of 20 rats undergoing cecal ligation to 20 with sham surgery, and analysing femoral bone strength using microCT, microscopy, and histological analysis is original and interesting. The findings of reduced fracture load, lower collagen elastic modulus, bone mineral elastic modulus, but no difference in BMD or bone architecture as measured by microCT, are interesting. Overall these results suggest early mechanisms other than loss of BMD may be important in critical illness, a plausible theory, particularly given the aetiology, risk factors, mechanisms, time course, and impact of altered bone health after critical illness remains largely unexplored.

Also, this study adds to the existing population based literature as there is a lack of microscopic or histological data exploring mechanisms of abnormal bone health after critical illness. As the authors point out it is difficult to obtain this in human, so laboratory data is vital. These results could lead to further research in longer term changes in bone morphology observed over time (i.e. do early changes result in microarchitectural change along term), and the effects of targeted interventions.

Many thanks for your kind words regarding our data. These data will indeed form the basis of interventional studies.

Reviewer 2: This is an animal study exploring an important area of emerging interest and provides some insights into the mechanism of early skeletal perturbations in critical illness providing a basis for further clinical studies.

It builds on recent work around the role of bone in the syndrome of critical illness. Their findings that bone architecture and BMD remained unchanged and there was no histological changes challenge some of the current understanding of the subject and merit further study.

The authors have adequately addressed reviewers' comments.

Thank you.

Reviewer 3:

C1: Overall I think this is a valuable and novel piece of literature. In my opinion, the authors have adequately address the comments from the first revision.

Thank you.

C2: How did the authors choose the sample size for the study and was a primary outcome selected a priori? I understand that it is not as "standard" for animal studies to consider this during the design. That said, animal studies suffer the same risks as human studies with respect to being underpowered to show differences and reporting bias. Along these same lines the authors should be careful in their wording about what they were able to prove as "Statistically different" vs. what appeared to be big difference between groups, but where they did not have adequate sample to show statistical difference given either measurement error or significant within animal variability (large SDs). They provide a long list of "not different" things in the discussion - The chance for type II error (in addition to type I) error is significant given the sample size.

We completely agree with the reviewer's comments re differences and reporting bias. Further, the chance of a type II error was not addressed, and we have added a paragraph to the discussion under limitations:

"Whilst no formal power calculation was performed, AFM scanning microscopy comparing bone stiffness in wild-type mice to those with osteogenesis imperfecta, has detected significant differences using a sample size of 21 . We expected a larger standard deviation in our CLP group, therefore a larger sample size was selected, consistent with other animal publications in critical illness2-4. Despite this, in humans patchy myonecrosis has been seen affecting different areas of muscle5- if the same were true for bone, histological and immunostaining data reported in this manuscript may be at risk of a Type II error." 
C3: I also wonder whether the authors should consider whether the animal model is truly one of "critical illness". Based upon the description provided in the methods and results it could be argued that the animals were not that unwell - they received minimal support following CLP and all lived. What would be expected when compared to other CLP papers? The fact all the animals lived should be discussed (at least in the limitations section). In my opinion some attention to this may support, instead of undermine, the importance of the findings. If the authors were able to identify significant functional changes on a model of "mild/moderate" critical illness, one can only imagine the differences they would have shown in a model where the animals were sick enough to require intubation, vasopressors, renal replacement, etc.

Thank you for this highly constructive comment. We have added:

"The CLP model used had a $0 \%$ rate of mortality, unlike the $55 \%$ reported in other $50 \%$ caecal ligation studies6. Therefore, this model is more likely to represent a $\mathrm{mild} / \mathrm{moderate}$ form of critical illness. Models with higher mortality and end-organ damage (perhaps more representative of the higher acuity spectrum of critical illness) may demonstrate greater loss of elastic modulus and bone strength."

C4: 3 . How did the authors choose 24 and 96 hours? Can they provide a justification? Is this long enough to expect to see changes (BMD and microarchitecture).

We thank the reviewer for this useful comment. 96 hours was chosen for two reasons. First, we were principally interested in early bone changes during critical illness.

Second, we actually expected mortality to approach 50 by 96 hours in the CLP group6. Therefore, we would have needed many more rodents in the CLP group to extend the study beyond 96 hours. We have added the following to the discussion to address this comment.

"Our animals were sacrificed at 96 hours, and it is possible insufficient time elapsed to accumulate changes in BMD or microarchitecture. However, rodent metabolic and muscle changes can be detected within this time frame7, and our hypothesis is focused on identifying bone changes in early critical illness, where interventions could modulate bone health in survivors."

C5: 4. All of the above factors intersect to work against the authors. Small sample size + non-critically ill animal model + limited time frame for outcome evaluation following insult. Again, this may work to the advantage of the authors as despite this they do show some interesting differences. These problems could be discussed in greater depth and framed in a light to support the need for larger studies on animal models or humans where it is possible to induce true critical illness requiring intervention, and is long lasting.

Thank you again for this constructive comment. We have added the following to the discussion, incorporating the response to comment 1 :

"Despite the lower acuity and limited time frame, differences in elastic modulus and bone strength were demonstrated. Whilst no formal power calculation was performed, AFM scanning microscopy comparing bone stiffness in wild-type mice to those with osteogenesis imperfecta, detected significant differences using a sample size of 21 . We expected a larger standard deviation in our CLP group, therefore a larger sample size was selected, consistent with other animal publications in critical illness2-4. Despite this, in humans, patchy myonecrosis has been seen affecting different areas of muscle5- if the same were true for bone, histological and immunostaining data reported in this manuscript may be at risk of a Type II error. Our data suggest the need for larger studies on animal models (induced with a higher acuity of critical illness) or human subjects where sustainable interventions can be additionally be assessed."

\section{REFERENCES}

1.Li T, Chang S-W, Rodriguez-Florez N, et al.: Studies of chain substitution caused sub-fibril level differences in stiffness and ultrastructure of wildtype and oim/oim collagen fibers using multifrequency-AFM and molecular modeling. Biomaterials 2016; 
107:15-22

2. Patel BV, Wilson MR, O'Dea KP, et al. TNF-induced death signaling triggers alveolar epithelial dysfunction in acute lung injury. J Immunol 2013;190(8):4274-82.

3. Files DC, D'Alessio FR, Johnston LF, et al. A critical role for muscle ring finger-1 in acute lung injury-associated skeletal muscle wasting. American journal of respiratory and critical care medicine 2012;185(8):825-34.

4. Files DC, Liu C, Pereyra A, et al. Therapeutic exercise attenuates neutrophilic lung injury and skeletal muscle wasting. Sci Transl Med 2015;7(278):278ra32.

5. Puthucheary ZA, Phadke R, Rawal J, et al. Qualitative Ultrasound in Acute Critical Illness Muscle Wasting. Critical care medicine 2015;43(8):1603-11.

6. Rittirsch D, Huber-Lang MS, Flierl MA, Ward PA. Immunodesign of experimental sepsis by cecal ligation and puncture. Nat Protoc. 2009;4:31-6.

7. O'Leary MJ, Ferguson CN, Rennie MJ, et al.: Sequential changes in in vivo muscle and liver protein synthesis and plasma and tissue glutamine levels in sepsis in the rat. Clin Sci 2001; 101:295-304 
Critical Care Medicine

500 Midway Drive

Mount Prospect, IL 60056

$16^{\text {th }}$ August 2017

Dear Prof. Buchman,

RE:Sepsis reduces bone strength before morphological changes are identifiable

Many thanks for the opportunity to further revise our manuscript. All points made by reviewer 3 have been addressed and the manuscript improved as a result-hopefully in a satisfactory manner to allow acceptance.

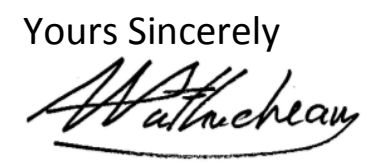

Dr Zudin Puthucheary (on behalf of the co-authors)

Zudin.puthucheary.09@ucl.ac.uk

Adult Intensive Care Unit

Royal Brompton Hospital

London WC1E 6AU

Mobile: 00447767357983

Tel: 00442076790840 


\section{Reply to Reviewers: Sepsis reduces bone strength}

Reviewer 1: This experimental study adds laboratory based evidence to the emerging literature on increased bone turnover, loss of bone density, and fracture after critical illness. The comparison of 20 rats undergoing cecal ligation to 20 with sham surgery, and analysing femoral bone strength using microCT, microscopy, and histological analysis is original and interesting. The findings of reduced fracture load, lower collagen elastic modulus, bone mineral elastic modulus, but no difference in BMD or bone architecture as measured by microCT, are interesting. Overall these results suggest early mechanisms other than loss of BMD may be important in critical illness, a plausible theory, particularly given the aetiology, risk factors, mechanisms, time course, and impact of altered bone health after critical illness remains largely unexplored.

Also, this study adds to the existing population based literature as there is a lack of microscopic or histological data exploring mechanisms of abnormal bone health after critical illness. As the authors point out it is difficult to obtain this in human, so laboratory data is vital. These results could lead to further research in longer term changes in bone morphology observed over time (i.e. do early changes result in microarchitectural change along term), and the effects of targeted interventions.

Many thanks for your kind words regarding our data. These data will indeed form the basis of interventional studies.

Reviewer 2: This is an animal study exploring an important area of emerging interest and provides some insights into the mechanism of early skeletal perturbations in critical illness providing a basis for further clinical studies.

It builds on recent work around the role of bone in the syndrome of critical illness. Their findings that bone architecture and BMD remained unchanged and there was no histological changes challenge some of the current understanding of the subject and merit further study. The authors have adequately addressed reviewers' comments.

Thank you.

Reviewer 3: 
C1: Overall I think this is a valuable and novel piece of literature. In my opinion, the authors have adequately address the comments from the first revision.

Thank you.

C2: How did the authors choose the sample size for the study and was a primary outcome selected a priori? I understand that it is not as "standard" for animal studies to consider this during the design. That said, animal studies suffer the same risks as human studies with respect to being underpowered to show differences and reporting bias. Along these same lines the authors should be careful in their wording about what they were able to prove as "Statistically different" vs. what appeared to be big difference between groups, but where they did not have adequate sample to show statistical difference given either measurement error or significant within animal variability (large SDs). They provide a long list of "not different" things in the discussion - The chance for type II error (in addition to type I) error is significant given the sample size.

We completely agree with the reviewer's comments re differences and reporting bias. Further, the chance of a type II error was not addressed, and we have added a paragraph to the discussion under limitations:

"Whilst no formal power calculation was performed, AFM scanning microscopy comparing bone stiffness in wild-type mice to those with osteogenesis imperfecta, has detected significant differences using a sample size of $2^{1}$. We expected a larger standard deviation in our CLP group, therefore a larger sample size was selected, consistent with other animal publications in critical illness ${ }^{2-4}$. Despite this, in humans patchy myonecrosis has been seen affecting different areas of muscle ${ }^{5}$ - if the same were true for bone, histological and immunostaining data reported in this manuscript may be at risk of a Type II error."

C3: I also wonder whether the authors should consider whether the animal model is truly one of "critical illness". Based upon the description provided in the methods and results it could be argued that the animals were not that unwell - they received minimal support following CLP and all lived. What would be expected when compared to other CLP papers? The fact all the animals lived should be discussed 
(at least in the limitations section). In my opinion some attention to this may support, instead of undermine, the importance of the findings. If the authors were able to identify significant functional changes on a model of "mild/moderate" critical illness, one can only imagine the differences they would have shown in a model where the animals were sick enough to require intubation, vasopressors, renal replacement, etc.

Thank you for this highly constructive comment. We have added:

"The CLP model used had a 0\% rate of mortality, unlike the 55\% reported in other $50 \%$ caecal ligation studies ${ }^{6}$. Therefore, this model is more likely to represent a mild/moderate form of critical illness. Models with higher mortality and end-organ damage (perhaps more representative of the higher acuity spectrum of critical illness) may demonstrate greater loss of elastic modulus and bone strength."

C4: 3. How did the authors choose 24 and 96 hours? Can they provide a justification? Is this long enough to expect to see changes (BMD and microarchitecture).

We thank the reviewer for this useful comment. 96 hours was chosen for two reasons. First, we were principally interested in early bone changes during critical illness. Second, we actually expected mortality to approach 50 by 96 hours in the CLP group ${ }^{6}$. Therefore, we would have needed many more rodents in the CLP group to extend the study beyond 96 hours. We have added the following to the discussion to address this comment.

"Our animals were sacrificed at 96 hours, and it is possible insufficient time elapsed to accumulate changes in BMD or microarchitecture. However, rodent metabolic and muscle changes can be detected within this time frame ${ }^{7}$, and our hypothesis is focused on identifying bone changes in early critical illness, where interventions could modulate bone health in survivors."

C5: 4. All of the above factors intersect to work against the authors. Small sample size + non-critically ill animal model + limited time frame for outcome evaluation following insult. Again, this may work to the advantage of the authors as despite this they do show some 
interesting differences. These problems could be discussed in greater depth and framed in a light to support the need for larger studies on animal models or humans where it is possible to induce true critical illness requiring intervention, and is long lasting.

Thank you again for this constructive comment. We have added the following to the discussion, incorporating the response to comment 1 :

"Despite the lower acuity and limited time frame, differences in elastic modulus and bone strength were demonstrated. Whilst no formal power calculation was performed, AFM scanning microscopy comparing bone stiffness in wild-type mice to those with osteogenesis imperfecta, detected significant differences using a sample size of $2^{1}$. We expected a larger standard deviation in our CLP group, therefore a larger sample size was selected, consistent with other animal publications in critical illness ${ }^{2-4}$. Despite this, in humans, patchy myonecrosis has been seen affecting different areas of muscle - if the same were true for bone, histological and immunostaining data reported in this manuscript may be at risk of a Type II error. Our data suggest the need for larger studies on animal models (induced with a higher acuity of critical illness) or human subjects where sustainable interventions can be additionally be assessed."

\section{REFERENCES}

1. Li T, Chang S-W, Rodriguez-Florez N, et al:: Studies of chain substitution caused sub-fibril level differences in stiffness and ultrastructure of wildtype and oim/oim collagen fibers using multifrequency-AFM and molecular modeling. Biomaterials 2016; 107:15-22

2. Patel BV, Wilson MR, O'Dea KP, et al. TNF-induced death signaling triggers alveolar epithelial dysfunction in acute lung injury. J Immunol 2013;190(8):4274-82.

3. Files DC, D'Alessio FR, Johnston LF, et al. A critical role for muscle ring finger-1 in acute lung injury-associated skeletal muscle wasting. American journal of respiratory and critical care medicine 2012;185(8):825-34.

4. Files DC, Liu C, Pereyra A, et al. Therapeutic exercise attenuates neutrophilic lung injury and skeletal muscle wasting. Sci Transl Med 2015;7(278):278ra32.

5. Puthucheary ZA, Phadke R, Rawal J, et al. Qualitative Ultrasound in Acute Critical Illness Muscle Wasting. Critical care medicine 2015;43(8):1603-11.

6. Rittirsch D, Huber-Lang MS, Flierl MA, Ward PA. Immunodesign of experimental sepsis by cecal ligation and puncture. Nat Protoc. 2009;4:31-6.

7. O'Leary MJ, Ferguson CN, Rennie MJ, et al.: Sequential changes in in vivo muscle and liver protein synthesis and plasma and tissue glutamine levels in sepsis in the rat. Clin Sci 2001; 101:295-304 

Zudin A. Puthucheary MBBS PhD ${ }^{1,2,3}$, Yao Sun BSc M.Eng ${ }^{4}$, Kaiyang Zeng PhD ${ }^{4}$,

\author{
Address:- 1. Department of Medicine, Yong Loo Lin School of \\ Medicine, National University Singapore, 1E Kent \\ Ridge Road, NUHS Tower Block Level 10, Singapore \\ 119228 \\ 2. Division of Critical Care, Royal Brompton Hospital, \\ London, UK \\ 3. Centre for Human Health and Performance, University \\ College London, UK \\ 4. Department of Mechanical Engineering, National \\ University Singapore, 9 Engineering Drive 1,Singapore \\ 117576 \\ * Both authors contributed equally to the manuscript
}

Corresponding author:- Zudin Puthucheary

Zudin.puthucheary.09@ucl.ac.uk

Adult Intensive Care Unit

Royal Brompton Hospital

United Kingdom

Mobile: 00447767357983

Tel: 00442076790840

Author Contributions:- ZP, conceptual design, analysis, interpretation, manuscript 
drafting, final approval and accountable for accuracy of data presented.

SY, LHV, ZZ, RZLL, data acquisition, analysis, manuscript revising, final approval and accountable for accuracy of data presented.

KZ, NSYC, MEC, conceptual design, analysis, interpretation, manuscript drafting, final approval and accountable for accuracy of data presented.

Funding support:- Medicine-Engineering seed grant from National University Health System and Faculty of Engineering at National University Singapore (R-265-000-532-112) (ZP, KZ, MEC).

Singapore National Medical Research Council Transitional Award funding, NMRC/TA/0015/2013 (MEC).

Ministry of Education (Singapore) Academic Research Grant (AcRF) (R-265-000-495-112) (KZ, YS).

Word count:- 2811 excluding abstract, legends, references. Abstract word count 250

Key words: Critical illness, Bone loss, Functional Disability 
2 Objectives: Survivors of critical illness have an increased incidence of bone fractures.

3 However, early changes in bone strength, and their relationship to structural changes,

4 have not been described. We aimed to characterize early changes in bone functional properties in critical illness and their relationship to changes in bone structure, using a

6 sepsis rodent model.

7 Design: Experimental Study

8 Setting: Animal Research Laboratory

9 Subjects: Adult Sprague-Dawley rats

10 Interventions: Forty Sprague-Dawley rats were randomised to cecal ligation and puncture (CLP) or sham surgery. Twenty rodents (10 CLP, 10 sham) were sacrificed 12 at 24 hours, and 20 more at 96 hours.

13 Measurements and main results: Femoral bones were harvested for strength testing, microCT imaging, histological analysis, and multi-frequency scanning probe microscopy (SPM). Fracture loads at the femoral neck were significantly reduced for CLP-exposed rodents at 24 hours $(83.39 \pm 10.1 \mathrm{~N}$ vs. $103.1 \pm 17.6 \mathrm{~N} ; \mathrm{p}=0.014)$ and 96

17 hours $(81.60 \pm 14.2 \mathrm{~N}$ vs. $95.66 \pm 14.3 \mathrm{~N} ; \mathrm{p}=0.047)$. Using multi-frequency $\mathrm{SPM}$, collagen elastic modulus was lower in CLP-exposed rats at 24 hours $(1.37 \pm 0.2 \mathrm{GPa}$ vs. $6.13 \pm 0.3 \mathrm{GPa} ; \mathrm{p}=0.001)$ and 96 hours $(5.57 \pm 0.5 \mathrm{GPa}$ vs. $6.13 \pm 0.3 \mathrm{GPa} ; \mathrm{p}=0.006)$. Bone mineral elastic modulus was similar at 24 hours, but reduced in CLP-exposed rodents

21 at 96 hours $(75.34 \pm 13.2 \mathrm{GPa}$ vs. $134.4 \pm 8.2 \mathrm{GPa} ; \mathrm{p}<0.001)$. There were no bone architectural or Bone Mineral Density differences by microCT. Similarly, histological analysis demonstrated no difference in collagen and, elastin staining, and Chemokine Receptor type 4, Nuclear Factor Kappa Beta and Tartarate Resistant Acid Phosphatase immunostaining. 
1 Conclusions: In a rodent sepsis model, trabecular bone strength is functionally

2 reduced within 24 hours and is associated with a reduction in collagen and mineral

3 elastic modulus. This is likely to be the result of altered biomechanical properties,

4 rather than increased bone mineral turnover. These data offer both mechanistic

5 insights and may potential guide development of therapeutic interventions. 
2 Intensive care unit (ICU) survivors suffer from a variety of cognitive, neurological 3 and physical impairments, which persist beyond acute care hospitalization and have

4 been described as the "Post-Intensive Care Syndrome" (PICS) (1). Bone health is 5 likely to be an important component of PICS (2), and population studies have 6 demonstrated a higher incidence of bone fractures in patients discharged from ICU 7 (3). Subsequent studies, seeking mechanistic explanations, have demonstrated 8 reduced bone mineral density (BMD) in ICU survivors $(4,5)$. However, it remains unclear whether the observed BMD changes, and increased fracture incidence, result from the direct effects of critical illness, prolonged immobility, or both (6).

Although it is clearly established that prolonged immobility contributes to much neuromuscular morbidity in ICU survivors $(7,8)$, it is also apparent that metabolic derangements and cytokinaemia in early critical illness play a pivotal role $(9,10)$. Similarly, critically ill patients are exposed to a variety of insults that may rapidly compromise bone structure and composition, such as inflammation, acidaemia, vitamin D deficiency, corticosteroid use and hypoxia (5). These adverse stimuli may result in structural changes, or alterations in bone turnover mediated by upregulation of pathways affecting osteoblastogenesis (11) or osteclastogenesis (12), or a combination. Therefore, bone health during, or following, critical illness may be the result of a number of factors, and complete understanding is likely to include mechanisms other than accumulated loss of BMD.

24 Factors beyond BMD certainly influence bone strength and susceptibility to fracture

25 (13). Indeed, bone displays a hierarchical organization in its structure and 
composition, ranging from macroscopic to molecular scales. Other, important, components of bone morphology influencing bone strength include bone size, cortical thickness and moment of inertia (14). In addition to whole bone morphology, bone microarchitecture, such as trabeculae shape and cortical porosity, as well as tissue properties, including collagen cross-linking and hydration, play important roles in bone strength. As a result, qualitative changes in bone strength and mechanical properties, independent of BMD, are well recognized (14).

We hypothesized that critical illness results in early, functionally significant, changes in bone strength. Since invasive bone studies are not feasible in critically ill patients (15), we used a rodent model of sepsis to investigate the effects of systemic sepsis and inflammation on mechanical bone strength; nearly $40 \%$ of critically ill patients admitted to the intensive care unit (ICU) are affected by sepsis (16). In addition, we conducted a histomorphometric analysis to identify macro- and microscopic perturbations that may offer mechanistic insight.

\section{METHODS}

Expanded methods available in online supplement.

\section{Rat sepsis model}

After obtaining Institutional Animal Care and Use Committee approval, 40 male Sprague-Dawley rats were randomized to receive cecal ligation and puncture (CLP) or sham surgery. In CLP rodents, $50 \%$ of the cecum was ligated and the anterior and posterior walls puncture with an $18 \mathrm{G}$ needle in a single pass (17). In sham rodents, cecum was mobilized and replaced. Following surgery, rats were given subcutaneous fluid and analgesia and returned to individual cages with food ad libitum. After 24 

and femur bones harvested. Non-invasive imaging was performed prior to strength

3 testing.

\section{$5 \quad$ Bone mechanical testing}

6 Prior to testing biomechanical properties, bone dimensions were measured used a

$7 \quad$ Vernier calliper.

8

9 Three-point bending test: To measure cortical bone biomechanical properties, the right femur underwent a 3-point bending analysis. Each bone sample was placed horizontally on two transverse supports (span length $(\mathrm{L}) 17 \mathrm{~mm}$ ) with the anterior surface facing up. Load was applied perpendicularly to the bone till fracture at a constant rate of $5 \mathrm{~mm} / \mathrm{min}$, using a materials testing machine, Instron-5543 (Instron Corp, Canton MA, USA). The parameters measured were load at break (N), Young's modulus (MPa), flexure stress at maximum load (MPa) and flexure strain (extension) at maximum load $(\mathrm{mm} / \mathrm{mm})$ normalized to outer thickness of bone.

Femoral neck break: To measure trabecular bone biomechanical properties, the femur underwent a femur neck break analysis. Following the 3-point bending test, samples were potted using dental cement and the length of neck and angle (Radian) measured.

21 Samples were clamped down and a vertical load, using a flat-surface arrow-head cylinder (Instron 5543, Instron Corp, Canton MA, USA), was applied at the top of the femoral head parallel to the axis of its diaphysis at a constant rate of $5 \mathrm{~mm} / \mathrm{min}$ till 
$2 \mathrm{~mm})$.

\section{$4 \quad$ Assessment of bone nano-mechanics}

5 Femurs were cleaned, sectioned, air dried, embedded in epoxy resin, mechanically

6 polished and placed on microscope slides. AM-FM (amplitude modulation-frequency

7 modulation) multi-frequency scanning probe microscopy (SPM) was then performed

8 (18).

9

10 An oscillating sharpened probe (connected to a cantilever) was moved over the 11 sample surface. Bone surface topography was mapped by cantilever movement, with 12 the spring-like action of the cantilever allowing force measurements to be performed 13 (19). The SPM probe was excited at two eigen-frequencies. The first eigenmode amplitude was used to image surface topography and the second eigenmode resonant frequency shift was used to map contact stiffness (20). Twenty measurements were taken from each bone sample (10 collagen, 10 mineral) with the average presented as a single data point for modulus in each sample.

\section{Micro-computed tomography measurements}

Femurs were scanned ex vivo using a Quantum GX micro-computerised tomography (microCT) imaging system (PerkinElmer, Waltham, Massachusetts, United States). Images were analysed at 3 locations, both at the shaft and neck, for bone volume/total volume $(\%)$, trabecular thickness $(\mathrm{mm})$, trabecular separation $(\mathrm{mm})$, connectivity density $\left(\mathrm{mm}^{-3}\right)$, degree of anisotropy (DA) and bone mineral density, (BMD) $\left(\mathrm{g} / \mathrm{mm}^{3}\right)$ using BoneJ software (21). 
2 Bone histomorphometry

3 Bones were measured using a Vernier caliper and then prepared for histological

4 staining. Slides were stained with hemotoxylin and eosin, Masson's trichrome stain

5 (for collagen) and Verhoeff stain (for elastin). The total number of osteoblasts within

6 a specified area on either side of the fracture was manually counted and a proportion

7 checked by an independent blinded histopathologist. Verhoeff and Masson trichrome

8 stained areas were analyzed using ImageJ (22) and results expressed as a percentage

9 of total bone area.

10

11 Immunohistochemistry was performed on deparaffinized bone sections using

12 the appropriate primary and secondary antibodies; tartrate-resistant acid phosphatase (TRAP) [ab58008] and Cysteine (C)-X-C motif chemokine receptor 4 (CXCR4) [ab124824] (Abcam, Cambridge, Massachusetts, USA); Nuclear Factor Kappa Beta (NF-кB) [\#8242] (Cell Signaling, Denver, Massachusetts, USA). Analysis was performed on tissue sections to quantify the number of positively stained cells per region of interest or per bone section using Image (22).

\section{Statistical analysis}

Data are presented as mean ( \pm standard deviation) or median (interquartile range) where appropriate. Differences were analyzed using one-way ANOVA with post hoc Bonferroni or Student's t-test for parametric data and Mann Whitney U test for nonparametric data. A p value $<0.05$ was considered significant. Levene's statistical test was used to compare distribution (standard deviation) data of elastic moduli. 
1

2 All forty rats survived to the end of the protocol. The weight at the time of surgery

3 was similar in all four groups (300 - 350g); weight loss between both groups was not

4 significantly different at 24 hours, but percentage weight loss was significantly greater

5 in the CLP-exposed group at 96 hours $(3.8 \%$ vs. $12.8 \% \mathrm{p}<0.01)$, as expected

6 (Supplemental Digital Content-Table 1).

7

8 Mechanical Bone Strength

9 Femoral neck: The maximum load required to fracture the femoral neck was $20 \%$ less for CLP-exposed rats relative to sham control after 24 hours $(83.39 \pm 10.1$ Newton $(\mathrm{N})$ vs. $103.1 \pm 17.6 \mathrm{~N} ; \mathrm{n}=8 ; \mathrm{p}=0.014)$. This difference persisted at 96 hours $(81.60 \pm 14.2 \mathrm{~N}$ vs. $95.66 \pm 14.3 \mathrm{~N} ; \mathrm{n}=9 ; \mathrm{P}=0.047$ ) (Figure 1A). Compressive strength was decreased in CLP-exposed rats at 24 hours, compared to sham $(44.32 \pm 5.7 \mathrm{~N}$ vs. $58.75 \pm 14.8 \mathrm{~N} ; \mathrm{n}=8$; $\mathrm{p}=0.021$ ); a similar difference was observed at 96 hours although not statistically significant $(45.8 \pm 12.1 \mathrm{~N}$ vs. $55.8 \pm 8.2 \mathrm{~N} ; \mathrm{n}=8 ; \mathrm{p}=0.068)$ (Figure $1 \mathrm{C})$. Bending strength differed in CLP-exposed rats, compared to sham, although statistical significance was achieved only at 96 hours (at 24 hours: $70.49 \pm 9.6 \mathrm{~N}$ vs. $83.87 \pm 15.9 \mathrm{~N} ; \mathrm{n}=8 ; \mathrm{p}=0.057$; at 96 hours: $66.89 \pm 12.3 \mathrm{~N}$ vs. $80.2 \pm 13.3 \mathrm{~N} ; \mathrm{n}=8 ; \mathrm{p}=0.038$ ) (Figure 1D).

Femoral shaft: There was no significant difference in the maximum load required to fracture the femoral shaft of CLP-exposed rats when compared to sham, at either 24 hours $(118.32 \pm 18.2 \mathrm{~N}$ vs. $119.78 \pm 23.9 \mathrm{~N} ; \mathrm{n}=10 ; \mathrm{p}=0.88)$ or 96 hours $(116.81 \pm 27.3 \mathrm{~N}$ vs. $128.75 \pm 28.5 .9 \mathrm{~N} ; \mathrm{n}=8 ; \mathrm{p}=0.379$ ) (Figure 1B). 
2 Collagen elastic modulus was lower after 24 hours in CLP rats compared to sham $3(1.37 \pm 0.2$ Gigapascals (GPa) vs. 6.13 $\pm 0.3 \mathrm{GPa} ; \mathrm{n}=8 ; \mathrm{p}=0.001)$. Despite partial 4 recovery at 96 hours, it remained lower than controls $(5.57 \pm 0.5 \mathrm{GPa}$ vs. $6.13 \pm 0.3$ $5 \mathrm{GPa} ; \mathrm{n}=8 ; \mathrm{p}=0.006$ ) (Figure $2 \mathrm{~A}$ ). In contrast, bone mineral elastic modulus was 6 similar in both groups at 24 hours $(128.7 \pm 8.1 \mathrm{GPa}$ vs. $134.4 \pm 8.2 \mathrm{GPa} ; \mathrm{n}=8 ; \mathrm{p}=0.131)$,

7 but reduced in CLP-exposed rats at 96 hours $(75.34 \pm 13.2 \mathrm{GPa}$ vs. 134.4 $\pm 8.2 \mathrm{GPa}$; $8 \mathrm{n}=8 ; \mathrm{p}<0.001$ ) (Figure 2B). Representative images are shown in Figure 3A and 3B.

9 The distributions of elastic moduli measurements for each group are shown in Figure

10 4. Collagen elastic modulus distribution was higher at 96 hours compared to sham 11 (Levene statistic 238.6, $\mathrm{p}<0.001$; Supplementary Digital Content Figure 1A) implying 12 impaired collagen quality recovery. The Mineral elastic modulus was similarly 13 affected at 96 hours (Levene statistic 150.5; p<0001; Supplementary Digital Content 14 Figure 1B).

\section{Whole bone geometry}

17 As shown in Supplemental Digital Content Table 2, there was no difference in cortical thickness, neck and shaft diameter or neck length between CLP and sham groups at 24 and 96 hours.

20

\section{Bone mineral density and microarchitecture}

Micro-CT reconstruction and histomorphometric analysis of the femoral neck did not reveal differences between CLP and sham in bone volume/total volume ratio, trabecular thickness and separation, connectivity density, degree of anisotropy and 
2 are shown in Figures 3C and 3D.

\section{$4 \quad$ Histological analysis}

5 Histologic sections demonstrated no difference in epiphyseal growth plate thickness

6 and cellular organization between CLP-exposed and sham groups at 24 hours

$7 \quad(118 \pm 7 \mu \mathrm{M}$ vs. $125 \pm 14 \mu \mathrm{M} ; \mathrm{p}=0.425)$ or 96 hours $(115 \pm 22 \mu \mathrm{M}$ vs. $102 \pm 6 \mu \mathrm{M}$;

$8 \mathrm{p}=0.289$ ). Osteoblast numbers did not differ in CLP-exposed rodents at 24 hours

$9(2.1 \pm 0.5$ vs. $2.2 \pm 0.8 ; \mathrm{p}=0.758)$ or at 96 hours $(1.8 \pm 0.4$ vs. $1.7 \pm 0.6 ; \mathrm{p}=0.684)$.

10 Similarly, no differences were seen in TRAP staining (marker of osteoclastic

11 differentiation) at 24 hours $(17.4 \pm 6.6 \%$ vs. $16.2 \pm 4.2 ; \mathrm{p}=0.803)$ and at 96 hours

$12(19.9 \pm 2.5 \%$ vs. $15.9 \pm 8.0 \% ; \mathrm{p}=0.456)$. No significant differences were seen between

13 both groups in percentage of elastin staining at 24 hours $(12.9 \pm 5.5 \%$ vs. $29.1 \pm 21.0 \%$;

$14 \mathrm{p}=0.185)$ or 96 hours $(34.0 \pm 22.8$ vs. $14.4 \pm 4.4 \% ; \mathrm{p}=0.143)$, and percentage of collagen staining $(75.6 \pm 11.5 \%$ vs. $70.1 \pm 17 \% \% ; \mathrm{p}=0.611)$ and $(70.5 \pm 15.0 \%$ vs. 75.0

$16 \pm 10.9 \% ; \mathrm{p}=0.638$ ). Representative images are shown in Figure 4.

CXCR4 staining did not differ between sham and CLP at 24 hours $(15.2 \pm 10.3 \%$ vs.

$11.6 \pm 5.0 \% ; \mathrm{p}=0.612)$ or 96 hours $(16.5 \pm 11.9 \%$ vs. $9.7 \pm 4.7 \% ; \mathrm{p}=0.407)$ and similarly with NF- $\mathrm{kB}$ at 24 hours $(2.2 \pm 1.4 \%$ vs. $3.3 \pm 1.1 \%$; $=0.325)$ and at 96 hours $(5.1 \pm 3.3 \%$ vs. $6.9 \pm 3.9 \%$; $=0.559$ ). Representative images are shown in Figure 5 . 


\section{DISCUSSION}

2

3 In this study, we set out to establish the nature of bone response to critical illness, and

4 the related functional consequences. In the CLP-exposed rodents there was evidence

5 of early functional changes, compared to sham surgery controls, with a lower

6 maximum load required to fracture the femoral neck at 24 hours. Multi-frequency

7 SPM demonstrated a rapid decrease in collagen elastic modulus at 24 hours, which

8 partially recovered at 96 hours. Mineral elastic modulus was preserved at 24 hours,

9 but decreased significantly at 96 hours.

10

11 Bone architecture and BMD remained unchanged, as determined by micro-CT.

12 Similarly, histological analysis revealed no differences in bone structure or collagen

13 and elastin content. The observed decrease in bone strength was not accompanied by any change in CXCR4 or NFא $\beta$ expression, or disruption in epiphyseal growth plate organization, osteoblast or osteoclast morphology or quantity and TRAP activity. The CXCR4 pathway plays a crucial role in the osteogenic differentiation of mesenchymal progenitors, and a disruption in its expression or function results in bone epiphyseal growth plate disorganization and abnormal steoblasts development (23). The transcription factor $\mathrm{NF} \kappa \beta$ is a crucial mediator of inflammatory responses, and has been implicated in promoting differentiation of myeloid cells into osteoclasts to exacerbate bone resorption, and to impair bone formation by disrupting osteoblast formation and function (24).

24 The observation of early functional changes to bone strength, in the absence of macroscopic or microscopic changes, reflect the complex organization of bone 
8 We used multi-frequency scanning probe microscopy (SPM) to analyze changes in 9

structure and the many factors contributing to bone strength. Alterations in matrix composition leading to loss of elastic modulus may effect bone strength, without changing BMD (25), but were excluded by histological analysis. Similarly, the lack of significant pertubations in osteoblast and osteoclast quantity and epiphyseal growth plate thickness and cellular organization, does not support increased bone turnover as an explanation for the early reduction in bone strength we observed.

bone tissue properties. This technique has been used to study the nano-mechanical properties of a range of biological tissues (26-28) including those of healthy and osteogenesis imperfecta bone $(28,29)$. In the absence of alterations in whole bone morphology, microarchitecture or histology, this nano-scale alteration in bone tissue properties may be an important determinant of the loss of bone mechanical strength $(14,30)$. Loss of mineral elastic modulus (and corresponding loss of stiffness) may account for the decrease in compression strength over time and loss of collagen elastic modulus (and loss of flexibility and tensile strength) for the reduction in bending strength.

The tissue properties of bone that determine bone strength include nature of the collagen, degree and type of collagen cross-linking, size and structure of hydroxyapatite crystals and degree of mineralization. The mineral component of bone is responsible for deformation resistance (31). In critically ill patients, circulating serum calcium has been observed to be associated with loss of bone mineral density acutely (5) and to normalize with recovery (4). This likely represents hydroxyapatite mobilization from mineral stores to maintain normocalcaemia (32). Loss of 
hydroxyapatite would therefore lead to loss of mineral elastic modulus, as seen in this rodent model of sepsis. Importantly, crystallinity modulation occurs independently of

3 bone tissue turnover (and NFk $\beta$ signaling) (30).

5 The properties of collagen fibers in bone tissue determine energy absorption, an 6 important component of fracture resistance (33). A recent summary of bone turnover 7 marker studies in critical illness (34) demonstrated the consistent increase in urinary 8 markers of loss of collagen mature cross-links - pyridinoline, deoxypyridinoline and collagen type $1 \mathrm{~N}$-Telopeptide $(4,35-39)$. Loss of collagen cross-link formation is associated with increased fracture risk in non-critical illness pathologies $(40,41)$. Alterations in cross-link formation would not be visible on histological analysis, explaining the lack of changes seen. The end result of loss of these essential intermolecular and interfibrillar cross-links, with likely associated altered collagen fibre orientation (40), would be both a weakening of the extracellular matrix, leading to reduced bending strength (42), and reduced elastic modulus, despite unchanged

16 bone mass (43).

Trabecular bone has a greater sensitivity to both processes than cortical bone $(44,45)$, leading to functional differences seen between the femoral neck and shaft in our study. Fractures of the femoral head constitute a major personal and public health issue and the loss of bone strength seen offers further biological plausibility to the observed acute and long-term increase in fracture risk described in survivors of critical illness (3-5). 


\section{Clinical Implications}

2 These data answer questions raised following Orford's seminal description of bone

3 loss in critical illness and its population level implications $(3,4)$. The rodents were not

4 exposed to glucocorticoids or sedation, yet both mineral and elastic modulus 5 decreased leading to a significant decrease in force needed to fracture.

6

7 The mechanism of increased bone fragility seems then to be the result of altered

8 biochemical properties of bone, as opposed to bone turnover driven loss of 9 mineralization. Thus, modulation of osteoclastogenesis e.g.(inhibition of Receptor 10 Activator for Nuclear Factor Kappa Beta) or osteoblastogeneisis (e.g. activation of 11 Transforming Growth Factor Beta) seems less likely to be effective mitigation 12 strategies. However, our data suggests that either bispohosphonate therapy (46) or 13 calcium normalization (47), to minimize hydroxyapatite mobilization ${ }_{2}$ may be 14 appropriate interventions. In addition, early mobilization and resistance exercise is likely to be of benefit in the clinical setting, increasing bone strength via alterations in 16 biomechanical properties (48), specifically collagen network organization and 17 deformation resistance, as opposed to increasing bone mineral density (49). Lastly, for 18 critical care survivors, smoking cessation therapy may have a specific role in bone 19 health (50).

\section{Limitations}

22 Our study does have important limitations to consider. Extrapolation of rodent data to humans cannot always be done with confidence. However, biological studies on bone 24 metabolism are challenging in humans, more so in the critical care setting. We thus

25 limited our research question to that of the fundamental bone biological response to a 
1 septic insult. The CLP model used had a $0 \%$ rate of mortality, unlike the $55 \%$

2 reported in $50 \%$ caecal ligation studiess $f^{7}(17)$ - Therefore, this model is more likely to

3 represent a mild/moderate form of critical illness. Models with higher mortality and

4 end-organ damage (perhaps more representative of the higher acuity spectrum of

5 critical illness) may demonstrate greater loss of elastic modulus and bone strength.

6 Our animals were sacrificed at 96 hours, and it is possible insufficient time elapsed to

7 accumulate changes in BMD or microarchitecture. However, rodent metabolic and

8 muscle changes can be detected within this time frame (51) and our hypothesis is

9 focused on identifying bone changes in early critical illness, where interventions

10 could modulate bone health in survivors. We did not measure circulating collagen

11 cross-link markers in the model, as this had been well described in humans, and our

12 focus was on altered biomechanical properties and mechanisms of such alterations.

13 Neither did we measure markers of advanced glycation end products which may

represent either Acute Lung Injury (52) or increased post-transcriptional modification

of new collagen fibres $(44,53,54)$. Future directions for animal model work might include exploration of therapies to modulate inorganic matrix mobilization and loss of collagen cross-links.

18

19 Despite the lower acuity and limited time frame, differences in elastic modulus and

20 bone strength were demonstrated. Whilst no formal power calculation was performed.

21 AFM scanning microscopy comparing bone stiffness in wild-type mice to those with osteogenesis imperfecta, has detected significant differences using a sample size of 2 
2 immunostaining data reported in this manuscript may be at risk of a Type II error.

3 Our data suggest the need for larger studies on animal models (induced with a higher

4 acuity of critical illness) or human subjects where sustainable interventions can be

5 additionally be assessed.

6

7 In conclusion, femoral neck strength is reduced in a rodent model of sepsis, with

8 associated decreases in both collagen and mineral modulus. The mechanism of this

9 phenomena is likely to be altered biomechanical properties instead of increased bone

10 turnover.

11

12 ACKNOWLEDGEMENTS

13 We acknowledge help and support with reading the histology slides from Dr Victor Lee MD PhD, Department of Pathology at National University Singapore.

15

16 Figure 1

17 Femur mechanical strength at neck (A) and shaft of femur (B). Compressive and 18 bending strength at the neck of femur is also shown ( $\mathrm{C}$ and $\mathrm{D}$, respectively). *Denotes data is significantly different from sham control subjects $(p<0.05)$. †Cecal ligation 20 and puncture. $\mathrm{N}=$ Newtons

Figure 2

23 (A) Collagen elastic modulus for sham, CLP(24) and CLP(96) groups. (B) Mineral 24 elastic modulus for sham, CLP(24) and CLP(96) groups. *Denotes data is 
2 puncture.

$4 \quad$ Figure 3

5 Representative images from multi-frequency scanning probe microscopy (SPM) and

6 microCT reconstruction. Multi-frequency SPM was used to image collagen (A) and

7 mineral (B) elastic modulus. MicroCT images were analysed in coronal (C) and

8 transverse (D) views.

9

$10 \quad$ Figure 4

11 Representative histology images showing results of hemotoxylin and eosin (H\&E)

12 staining, as well as staining for collagen (Masson's trichrome stain) and elastin

13 (Verhoeff stain). Scale bar is $100 \mu \mathrm{M}$.

Figure 5

16 Immunohistochemistry images using antibody staining for Chemokine Receptor type

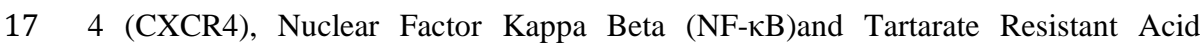
18 Phosphatase (TRAP) at the femoral neck. Scale bar is $100 \mu \mathrm{M}$.

19

\section{References}

22 1. Elliott D, Davidson JE, Harvey MA, et al.: Exploring the scope of postintensive care syndrome therapy and care: engagement of non-critical care providers and survivors in a second stakeholders meeting. Critical Care 
2. Griffith DM, Walsh TS: Bone loss during critical illness: a skeleton in the closet for the intensive care unit survivor? Critical Care Medicine 2011; 39:1554-1556

43 . Orford NR, Saunders K, Merriman E, et al.: Skeletal morbidity among survivors of critical illness. Critical Care Medicine 2011; 39:1295-1300

6 4. Orford NR, Lane SE, Bailey M, et al.: Changes in Bone Mineral Density in the Year after Critical Illness. Am J Respir Crit Care Med 2016; 193:736-744

8 5. Rawal J, McPhail MJW, Ratnayake G, et al.: A pilot study of change in fracture risk in patients with acute respiratory distress syndrome. Crit Care $2015 ; 19: 165$

6. Bilezikian JP: Bone Loss in the Intensive Care Unit. Am J Respir Crit Care Med 2016; 193:706-707

7. Fletcher SN, Kennedy DD, Ghosh IR, et al.: Persistent neuromuscular and neurophysiologic abnormalities in long-term survivors of prolonged critical

9. Kress JP, Hall JB: ICU-acquired weakness and recovery from critical illness. $N$ Engl J Med 2014; 370:1626-1635

10. Puthucheary ZA, Rawal J, McPhail M, et al.: Acute skeletal muscle wasting in

11. Chang J, Wang Z, Tang E, et al.: Inhibition of osteoblastic bone formation by nuclear factor-kappaB. Nat Med 2009; 15:682-689 
pathways in fracture repair. Bonekey Rep 2013; 2:300

2 13. Keaveny TM, Kopperdahl DL, Melton LJ, et al.: Age-dependence of femoral strength in white women and men. J Bone Miner Res 2010; 25:994-1001

4 14. Fonseca H, Moreira-Gonçalves D, Coriolano H-JA, et al.: Bone Quality: The Determinants of Bone Strength and Fragility. Sports Med 2013; 44:37-53

15. Elkin SL, Vedi S, Bord S, et al.: Histomorphometric analysis of bone biopsies from the iliac crest of adults with cystic fibrosis. Am J Respir Crit Care Med $2002 ; 166: 1470-1474$

16. Vincent J-L, Sakr Y, Sprung CL, et al.: Sepsis in European intensive care units: Results of the SOAP study*. Critical Care Medicine 2006; 34:344-353

17. Rittirsch D, Huber-Lang MS, Flierl MA, et al.: Immunodesign of experimental sepsis by cecal ligation and puncture. Nat Protoc 2009; 4:31-36

18. Garcia R, Herruzo ET: The emergence of multifrequency force microscopy. Nature Nanotechnology 2012; 7:217-226

19. Wallace JM: Applications of atomic force microscopy for the assessment of nanoscale morphological and mechanical properties of bone. Bone 2012; $50: 420-427$

20. Stark RW: Dynamics of repulsive dual-frequency atomic force microscopy. Applied Physics Letters 2009; 94:063109

21. Doube M, Kłosowski MM, Arganda-Carreras I, et al.: BoneJ: Free and extensible bone image analysis in ImageJ. Bone 2010; 47:1076-1079

22. Schneider CA, Rasband WS, Eliceiri KW: NIH Image to ImageJ: 25 years of image analysis. Nat Methods 2012; 9:671-675

23. Zhu W, Liang G, Huang Z, et al.: Conditional inactivation of the CXCR4 receptor in osteoprecursors reduces postnatal bone formation due to impaired 
24. Swarnkar G, Zhang K, Mbalaviele G, et al.: Constitutive activation of IKK2/NF-кB impairs osteogenesis and skeletal development. PLoS ONE 2014; 9:e91421

25. Burr DB, Robling AG, Turner CH: Effects of biomechanical stress on bones in animals. Bone 2002; 30:781-786

26. Hansma HG, Kim KJ, Laney DE, et al.: Properties of biomolecules measured from atomic force microscope images: a review. J Struct Biol 1997; 119:99108

27. Hilal N, Bowen WR, Alkhatib L, et al.: A Review of Atomic Force Microscopy Applied to Cell Interactions with Membranes. Chemical Engineering Research and Design 2006; 84:282-292 
properties of collagen. J Struct Biol 1998; 122:119-122

2 34. Lee P, Nair P, Eisman JA, et al.: Bone Failure in Critical Illness. Critical Care Medicine 2016; 44:2270-2274

35. Smith LM, Cuthbertson B, Harvie J, et al.: Increased bone resorption in the critically ill: association with sepsis and increased nitric oxide production. Critical Care Medicine 2002; 30:837-840

36. Van den Berghe G, Baxter RC, Weekers F, et al.: The combined administration of GH-releasing peptide-2 (GHRP-2), TRH and GnRH to men with prolonged critical illness evokes superior endocrine and metabolic effects compared to treatment with GHRP-2 alone. Clin Endocrinol (Oxf) 2002; 56:655-669

37. Van den Berghe G, Van Roosbroeck D, Vanhove P, et al.: Bone turnover in prolonged critical illness: effect of vitamin D. J Clin Endocrinol Metab 2003; $88: 4623-4632$

38. Nierman DM, Mechanick JI: Bone hyperresorption is prevalent in chronically critically ill patients. Chest $1998 ; 114: 1122-1128$

39. Nierman DM, Mechanick JI: Biochemical response to treatment of bone hyperresorption in chronically critically ill patients. Chest 2000; 118:761-766

40. Martin E, Shapiro JR: Osteogenesis imperfecta: Epidemiology and pathophysiology. Current Osteoporosis Reports 2009; 5:91-97

41. Simon JA, Hudes ES: Relation of ascorbic acid to bone mineral density and self-reported fractures among US adults. Am J Epidemiol 2001; 154:427-433

42. Bailey AJ, Sims TJ, Ebbesen EN, et al.: Age-related changes in the biochemical properties of human cancellous bone collagen: relationship to bone strength. Calcified Tissue International 1999; 65:203-210

43. Banse X, Sims TJ, Bailey AJ: Mechanical properties of adult vertebral 
cancellous bone: correlation with collagen intermolecular cross-links. J Bone Miner Res 2002; 17:1621-1628

44. Viguet-Carrin S, Follet H, Gineyts E, et al.: Association between collagen cross-links and trabecular microarchitecture properties of human vertebral bone. Bone 2010; 46:342-347

45. Karim L, Tang SY, Sroga GE, et al.: Differences in non-enzymatic glycation and collagen cross-links between human cortical and cancellous bone. Osteoporos Int 2013; 24:2441-2447

46. Aris RM, Lester GE, Caminiti M, et al.: Efficacy of alendronate in adults with cystic fibrosis with low bone density. Am J Respir Crit Care Med 2004; $169: 77-82$

47. Worth H, Stammen D, Keck E: Therapy of steroid-induced bone loss in adult asthmatics with calcium, vitamin D, and a diphosphonate. Am J Respir Crit Care Med 1994; 150:394-397

48. Huang T-H, Chang F-L, Lin S-C, et al.: Endurance treadmill running training benefits the biomaterial quality of bone in growing male Wistar rats. $J$ Bone Miner Metab 2008; 26:350-357

49. Shiiba M, Arnaud SB, Tanzawa H, et al.: Regional alterations of type I collagen in rat tibia induced by skeletal unloading. J Bone Miner Res 2002; $17: 1639-1645$

50. Terashima T, Wiggs B, English D, et al.: The effect of cigarette smoking on the bone marrow. Am J Respir Crit Care Med 1997; 155:1021-1026

51. O'Leary MJ, Ferguson CN, Rennie MJ, et al.: Sequential changes in in vivo muscle and liver protein synthesis and plasma and tissue glutamine levels in sepsis in the rat. Clin Sci 2001; 101:295-304 
52. Calfee CS, Ware LB, Eisner MD, et al.: Plasma receptor for advanced glycation end products and clinical outcomes in acute lung injury. Thorax 2008; 63:1083-1089

53. Saito M, Marumo K: Collagen cross-links as a determinant of bone quality: a possible explanation for bone fragility in aging, osteoporosis, and diabetes mellitus. Osteoporos Int 2010; 21:195-214

54. Santana RB, $\mathrm{Xu} \mathrm{L}$, Chase $\mathrm{HB}$, et al.: A role for advanced glycation end products in diminished bone healing in type 1 diabetes. Diabetes 2003; 52:1502-1510

55. Patel BV, Wilson MR, O'Dea KP, et al.: TNF-induced death signaling triggers alveolar epithelial dysfunction in acute lung injury. J Immunol 2013; $190: 4274-4282$

56. Files DC, D'Alessio FR, Johnston LF, et al.: A critical role for muscle ring finger-1 in acute lung injury-associated skeletal muscle wasting. Am J Respir Crit Care Med 2012; 185:825-834

57. Files DC, Liu C, Pereyra A, et al.: Therapeutic exercise attenuates neutrophilic lung injury and skeletal muscle wasting. Sci Transl Med 2015; 7:278ra32

58. Puthucheary ZA, Phadke R, Rawal J, et al.: Qualitative Ultrasound in Acute Critical Illness Muscle Wasting. Critical Care Medicine 2015; 43:1603-1611 
1 Sepsis reduces bone strength before morphological changes are identifiable

2 Zudin A. Puthucheary MBBS PhD ${ }^{1,2,3}$, Yao Sun BSc M.Eng ${ }^{4}$, Kaiyang Zeng $\mathrm{PhD}^{4}$, 3 Lien Hong Vu PhD ${ }^{1}$, Zhi Wei Zhang BSc ${ }^{1}$, Ryan ZL Lim BSc ${ }^{1}$, Nicholas SY Chew ${ }^{*}$ MBChB $\mathrm{PhD}^{1}$, Matthew E. Cove ${ }^{*} \mathrm{MBChB}^{1}$. 5

\author{
Address:- 1 . Department of Medicine, Yong Loo Lin School of \\ Medicine, National University Singapore, 1E Kent \\ Ridge Road, NUHS Tower Block Level 10, Singapore \\ 119228
}

2. Division of Critical Care, Royal Brompton Hospital, London, UK

3. Centre for Human Health and Performance, University College London, UK

4. Department of Mechanical Engineering, National University Singapore, 9 Engineering Drive 1,Singapore 117576

* Both authors contributed equally to the manuscript

Corresponding author:- Zudin Puthucheary

Zudin.puthucheary.09@ucl.ac.uk

Adult Intensive Care Unit

Royal Brompton Hospital

United Kingdom

Mobile: 00447767357983

Tel: 00442076790840

Author Contributions:- ZP, conceptual design, analysis, interpretation, manuscript 
drafting, final approval and accountable for accuracy of data presented.

SY, LHV, ZZ, RZLL, data acquisition, analysis, manuscript revising, final approval and accountable for accuracy of data presented.

KZ, NSYC, MEC, conceptual design, analysis, interpretation, manuscript drafting, final approval and accountable for accuracy of data presented.

Funding support:- Medicine-Engineering seed grant from National University Health System and Faculty of Engineering at National University Singapore (R-265-000-532-112) (ZP, KZ, MEC).

Singapore National Medical Research Council Transitional Award funding, NMRC/TA/0015/2013 (MEC).

Ministry of Education (Singapore) Academic Research Grant (AcRF) (R-265-000-495-112) (KZ, YS).

Word count:- 2811 excluding abstract, legends, references. Abstract word count 250 .

Key words: Critical illness, Bone loss, Functional Disability 


\section{$1 \quad$ Abstract}

2 Objectives: Survivors of critical illness have an increased incidence of bone fractures.

3 However, early changes in bone strength, and their relationship to structural changes,

4 have not been described. We aimed to characterize early changes in bone functional

5 properties in critical illness and their relationship to changes in bone structure, using a

6 sepsis rodent model.

7 Design: Experimental Study

8 Setting: Animal Research Laboratory

9 Subjects: Adult Sprague-Dawley rats

10 Interventions: Forty Sprague-Dawley rats were randomised to cecal ligation and 11 puncture (CLP) or sham surgery. Twenty rodents (10 CLP, 10 sham) were sacrificed 12 at 24 hours, and 20 more at 96 hours.

13 Measurements and main results: Femoral bones were harvested for strength testing, 14 microCT imaging, histological analysis, and multi-frequency scanning probe 15 microscopy (SPM). Fracture loads at the femoral neck were significantly reduced for 16 CLP-exposed rodents at 24 hours $(83.39 \pm 10.1 \mathrm{~N}$ vs. $103.1 \pm 17.6 \mathrm{~N} ; \mathrm{p}=0.014)$ and 96 17 hours $(81.60 \pm 14.2 \mathrm{~N}$ vs. $95.66 \pm 14.3 \mathrm{~N} ; \mathrm{p}=0.047)$. Using multi-frequency SPM, 18 collagen elastic modulus was lower in CLP-exposed rats at 24 hours $(1.37 \pm 0.2 \mathrm{GPa}$ vs. $196.13 \pm 0.3 \mathrm{GPa} ; \mathrm{p}=0.001)$ and 96 hours $(5.57 \pm 0.5 \mathrm{GPa}$ vs. $6.13 \pm 0.3 \mathrm{GPa} ; \mathrm{p}=0.006)$. Bone 20 mineral elastic modulus was similar at 24 hours, but reduced in CLP-exposed rodents 21 at 96 hours $(75.34 \pm 13.2 \mathrm{GPa}$ vs. $134.4 \pm 8.2 \mathrm{GPa} ; \mathrm{p}<0.001)$. There were no bone 22 architectural or Bone Mineral Density differences by microCT. Similarly, histological 23 analysis demonstrated no difference in collagen and, elastin staining, and Chemokine 24 Receptor type 4, Nuclear Factor Kappa Beta and Tartarate Resistant Acid Phosphatase 25 immunostaining. 
1 Conclusions: In a rodent sepsis model, trabecular bone strength is functionally

2 reduced within 24 hours and is associated with a reduction in collagen and mineral

3 elastic modulus. This is likely to be the result of altered biomechanical properties,

4 rather than increased bone mineral turnover. These data offer both mechanistic

5 insights and may potential guide development of therapeutic interventions.

6 


\section{INTRODUCTION}

2 Intensive care unit (ICU) survivors suffer from a variety of cognitive, neurological

3 and physical impairments, which persist beyond acute care hospitalization and have

4 been described as the "Post-Intensive Care Syndrome" (PICS) (1). Bone health is

5 likely to be an important component of PICS (2), and population studies have

6 demonstrated a higher incidence of bone fractures in patients discharged from ICU

7 (3). Subsequent studies, seeking mechanistic explanations, have demonstrated

8 reduced bone mineral density (BMD) in ICU survivors $(4,5)$. However, it remains

9 unclear whether the observed BMD changes, and increased fracture incidence, result

10 from the direct effects of critical illness, prolonged immobility, or both (6).

12 Although it is clearly established that prolonged immobility contributes to much

13 neuromuscular morbidity in ICU survivors $(7,8)$, it is also apparent that metabolic

14 derangements and cytokinaemia in early critical illness play a pivotal role $(9,10)$.

15 Similarly, critically ill patients are exposed to a variety of insults that may rapidly

16 compromise bone structure and composition, such as inflammation, acidaemia,

17 vitamin D deficiency, corticosteroid use and hypoxia (5). These adverse stimuli may

18 result in structural changes, or alterations in bone turnover mediated by upregulation

19 of pathways affecting osteoblastogenesis (11) or osteclastogenesis (12), or a

20 combination. Therefore, bone health during, or following, critical illness may be the

21 result of a number of factors, and complete understanding is likely to include

22 mechanisms other than accumulated loss of BMD.

24 Factors beyond BMD certainly influence bone strength and susceptibility to fracture

25 (13). Indeed, bone displays a hierarchical organization in its structure and 
1 composition, ranging from macroscopic to molecular scales. Other, important,

2 components of bone morphology influencing bone strength include bone size, cortical

3 thickness and moment of inertia (14). In addition to whole bone morphology, bone

4 microarchitecture, such as trabeculae shape and cortical porosity, as well as tissue

5 properties, including collagen cross-linking and hydration, play important roles in

6 bone strength. As a result, qualitative changes in bone strength and mechanical

7 properties, independent of BMD, are well recognized (14).

8

9 We hypothesized that critical illness results in early, functionally significant, changes

10 in bone strength. Since invasive bone studies are not feasible in critically ill patients

11 (15), we used a rodent model of sepsis to investigate the effects of systemic sepsis and

12 inflammation on mechanical bone strength; nearly $40 \%$ of critically ill patients

13 admitted to the intensive care unit (ICU) are affected by sepsis (16). In addition, we

14 conducted a histomorphometric analysis to identify macro- and microscopic

15 perturbations that may offer mechanistic insight.

\section{METHODS}

18 Expanded methods available in online supplement.

\section{Rat sepsis model}

20 After obtaining Institutional Animal Care and Use Committee approval, 40 male

21 Sprague-Dawley rats were randomized to receive cecal ligation and puncture (CLP)

22 or sham surgery. In CLP rodents, $50 \%$ of the cecum was ligated and the anterior and

23 posterior walls puncture with an $18 \mathrm{G}$ needle in a single pass (17). In sham rodents,

24 cecum was mobilized and replaced. Following surgery, rats were given subcutaneous

25 fluid and analgesia and returned to individual cages with food ad libitum. After 24 
1 and 96 hours, 10 rats in each group were euthanized with carbon dioxide inhalation

2 and femur bones harvested. Non-invasive imaging was performed prior to strength

3 testing.

\section{Bone mechanical testing}

6 Prior to testing biomechanical properties, bone dimensions were measured used a

$7 \quad$ Vernier calliper.

9 Three-point bending test: To measure cortical bone biomechanical properties, the 10 right femur underwent a 3-point bending analysis. Each bone sample was placed 11 horizontally on two transverse supports (span length (L) $17 \mathrm{~mm}$ ) with the anterior 12 surface facing up. Load was applied perpendicularly to the bone till fracture at a 13 constant rate of $5 \mathrm{~mm} / \mathrm{min}$, using a materials testing machine, Instron-5543 (Instron 14 Corp, Canton MA, USA). The parameters measured were load at break (N), Young's 15 modulus (MPa), flexure stress at maximum load (MPa) and flexure strain (extension)

16 at maximum load $(\mathrm{mm} / \mathrm{mm})$ normalized to outer thickness of bone.

18 Femoral neck break: To measure trabecular bone biomechanical properties, the femur 19 underwent a femur neck break analysis. Following the 3-point bending test, samples 20 were potted using dental cement and the length of neck and angle (Radian) measured.

21 Samples were clamped down and a vertical load, using a flat-surface arrow-head 22 cylinder (Instron 5543, Instron Corp, Canton MA, USA), was applied at the top of the 23 femoral head parallel to the axis of its diaphysis at a constant rate of $5 \mathrm{~mm} / \mathrm{min}$ till 24 fracture. Parameters measured were maximum Load (N), Young's modulus (N/mm), 
1 compression component $(\mathrm{N})$, bending component $(\mathrm{N})$ and bending momentum $(\mathrm{N}-$

$2 \mathrm{~mm})$.

4 Assessment of bone nano-mechanics

5 Femurs were cleaned, sectioned, air dried, embedded in epoxy resin, mechanically

6 polished and placed on microscope slides. AM-FM (amplitude modulation-frequency

7 modulation) multi-frequency scanning probe microscopy (SPM) was then performed

8 (18).

9

10 An oscillating sharpened probe (connected to a cantilever) was moved over the

11 sample surface. Bone surface topography was mapped by cantilever movement, with

12 the spring-like action of the cantilever allowing force measurements to be performed

13 (19). The SPM probe was excited at two eigen-frequencies. The first eigenmode

14 amplitude was used to image surface topography and the second eigenmode resonant

15 frequency shift was used to map contact stiffness (20). Twenty measurements were

16 taken from each bone sample (10 collagen, 10 mineral) with the average presented as

17 a single data point for modulus in each sample.

19 Micro-computed tomography measurements

20 Femurs were scanned ex vivo using a Quantum GX micro-computerised tomography

21 (microCT) imaging system (PerkinElmer, Waltham, Massachusetts, United States).

22 Images were analysed at 3 locations, both at the shaft and neck, for bone volume/total

23 volume $(\%)$, trabecular thickness $(\mathrm{mm})$, trabecular separation $(\mathrm{mm})$, connectivity

24 density $\left(\mathrm{mm}^{-3}\right)$, degree of anisotropy (DA) and bone mineral density, (BMD) $\left(\mathrm{g} / \mathrm{mm}^{3}\right)$

25 using BoneJ software (21). 


\section{Bone histomorphometry}

3 Bones were measured using a Vernier caliper and then prepared for histological

4 staining. Slides were stained with hemotoxylin and eosin, Masson's trichrome stain

5 (for collagen) and Verhoeff stain (for elastin). The total number of osteoblasts within

6 a specified area on either side of the fracture was manually counted and a proportion

7 checked by an independent blinded histopathologist. Verhoeff and Masson trichrome

8 stained areas were analyzed using ImageJ (22) and results expressed as a percentage

9 of total bone area.

11 Immunohistochemistry was performed on deparaffinized bone sections using 12 the appropriate primary and secondary antibodies; tartrate-resistant acid 13 phosphatase (TRAP) [ab58008] and Cysteine (C)-X-C motif chemokine receptor 144 (CXCR4) [ab124824] (Abcam, Cambridge, Massachusetts, USA); Nuclear Factor 15 Kappa Beta (NF-кB) [\#8242] (Cell Signaling, Denver, Massachusetts, USA). 16 Analysis was performed on tissue sections to quantify the number of positively 17 stained cells per region of interest or per bone section using ImageJ (22).

\section{Statistical analysis}

20 Data are presented as mean ( \pm standard deviation) or median (interquartile range) 21 where appropriate. Differences were analyzed using one-way ANOVA with post hoc 22 Bonferroni or Student's t-test for parametric data and Mann Whitney U test for non23 parametric data. A p value $<0.05$ was considered significant. Levene's statistical test 24 was used to compare distribution (standard deviation) data of elastic moduli. 


\section{$1 \quad$ RESULTS}

2 All forty rats survived to the end of the protocol. The weight at the time of surgery

3 was similar in all four groups $(300-350 \mathrm{~g})$; weight loss between both groups was not

4 significantly different at 24 hours, but percentage weight loss was significantly greater

5 in the CLP-exposed group at 96 hours $(3.8 \%$ vs. $12.8 \% \mathrm{p}<0.01)$, as expected 6 (Supplemental Digital Content-Table 1).

7

\section{Mechanical Bone Strength}

9 Femoral neck: The maximum load required to fracture the femoral neck was $20 \%$ less

10 for CLP-exposed rats relative to sham control after 24 hours $(83.39 \pm 10.1$ Newton $(\mathrm{N})$

11 vs. $103.1 \pm 17.6 \mathrm{~N} ; \mathrm{n}=8 ; \mathrm{p}=0.014)$. This difference persisted at 96 hours $(81.60 \pm 14.2 \mathrm{~N}$

12 vs. $95.66 \pm 14.3 \mathrm{~N} ; \mathrm{n}=9 ; \mathrm{P}=0.047$ ) (Figure 1A). Compressive strength was decreased in

13 CLP-exposed rats at 24 hours, compared to sham $(44.32 \pm 5.7 \mathrm{~N}$ vs. $58.75 \pm 14.8 \mathrm{~N} ; \mathrm{n}=8$;

$14 \mathrm{p}=0.021$ ); a similar difference was observed at 96 hours although not statistically

15 significant $(45.8 \pm 12.1 \mathrm{~N}$ vs. $55.8 \pm 8.2 \mathrm{~N} ; \mathrm{n}=8 ; \mathrm{p}=0.068)$ (Figure $1 \mathrm{C})$. Bending strength

16 differed in CLP-exposed rats, compared to sham, although statistical significance was

17 achieved only at 96 hours (at 24 hours: $70.49 \pm 9.6 \mathrm{~N}$ vs. $83.87 \pm 15.9 \mathrm{~N} ; \mathrm{n}=8 ; \mathrm{p}=0.057$;

18 at 96 hours: $66.89 \pm 12.3 \mathrm{~N}$ vs. $80.2 \pm 13.3 \mathrm{~N} ; \mathrm{n}=8 ; \mathrm{p}=0.038$ ) (Figure $1 \mathrm{D}$ ).

19

20 Femoral shaft: There was no significant difference in the maximum load required to

21 fracture the femoral shaft of CLP-exposed rats when compared to sham, at either 24

22 hours $(118.32 \pm 18.2 \mathrm{~N}$ vs. $119.78 \pm 23.9 \mathrm{~N} ; \mathrm{n}=10 ; \mathrm{p}=0.88)$ or 96 hours $(116.81 \pm 27.3 \mathrm{~N}$

23 vs. $128.75 \pm 28.5 .9 \mathrm{~N} ; \mathrm{n}=8 ; \mathrm{p}=0.379$ ) (Figure $1 \mathrm{~B})$. 


\section{Bone nano-mechanics with multi-frequency scanning probe microscopy}

2 Collagen elastic modulus was lower after 24 hours in CLP rats compared to sham

3 (1.37 \pm 0.2 Gigapascals $(\mathrm{GPa})$ vs. $6.13 \pm 0.3 \mathrm{GPa} ; \mathrm{n}=8 ; \mathrm{p}=0.001)$. Despite partial

4 recovery at 96 hours, it remained lower than controls $(5.57 \pm 0.5 \mathrm{GPa}$ vs. $6.13 \pm 0.3$

$5 \mathrm{GPa} ; \mathrm{n}=8 ; \mathrm{p}=0.006$ ) (Figure 2A). In contrast, bone mineral elastic modulus was

6 similar in both groups at 24 hours $(128.7 \pm 8.1 \mathrm{GPa}$ vs. $134.4 \pm 8.2 \mathrm{GPa} ; \mathrm{n}=8 ; \mathrm{p}=0.131)$,

7 but reduced in CLP-exposed rats at 96 hours $(75.34 \pm 13.2 \mathrm{GPa}$ vs. $134.4 \pm 8.2 \mathrm{GPa}$;

$8 \mathrm{n}=8 ; \mathrm{p}<0.001$ ) (Figure 2B). Representative images are shown in Figure 3A and 3B.

9 The distributions of elastic moduli measurements for each group are shown in Figure

10 4. Collagen elastic modulus distribution was higher at 96 hours compared to sham

11 (Levene statistic 238.6, $\mathrm{p}<0.001$; Supplementary Digital Content Figure 1A) implying 12 impaired collagen quality recovery. The Mineral elastic modulus was similarly

13 affected at 96 hours (Levene statistic 150.5; p<0001; Supplementary Digital Content 14 Figure 1B).

16 Whole bone geometry

17 As shown in Supplemental Digital Content Table 2, there was no difference in cortical 18 thickness, neck and shaft diameter or neck length between CLP and sham groups at 1924 and 96 hours.

\section{Bone mineral density and microarchitecture}

22 Micro-CT reconstruction and histomorphometric analysis of the femoral neck did not

23 reveal differences between CLP and sham in bone volume/total volume ratio, 24 trabecular thickness and separation, connectivity density, degree of anisotropy and 
1 bone mineral density at 24 or 96 hours (all $p>0.10$, Table S1). Representative images

2 are shown in Figures 3C and 3D.

3

\section{$4 \quad$ Histological analysis}

5 Histologic sections demonstrated no difference in epiphyseal growth plate thickness

6 and cellular organization between CLP-exposed and sham groups at 24 hours $7 \quad(118 \pm 7 \mu \mathrm{M}$ vs. $125 \pm 14 \mu \mathrm{M} ; \mathrm{p}=0.425)$ or 96 hours $(115 \pm 22 \mu \mathrm{M}$ vs. $102 \pm 6 \mu \mathrm{M}$; $8 \mathrm{p}=0.289$ ). Osteoblast numbers did not differ in CLP-exposed rodents at 24 hours $9(2.1 \pm 0.5$ vs. $2.2 \pm 0.8 ; \mathrm{p}=0.758)$ or at 96 hours $(1.8 \pm 0.4$ vs. $1.7 \pm 0.6 ; \mathrm{p}=0.684)$. 10 Similarly, no differences were seen in TRAP staining (marker of osteoclastic 11 differentiation) at 24 hours $(17.4 \pm 6.6 \%$ vs. $16.2 \pm 4.2 ; \mathrm{p}=0.803)$ and at 96 hours $12(19.9 \pm 2.5 \%$ vs. $15.9 \pm 8.0 \% ; \mathrm{p}=0.456)$. No significant differences were seen between 13 both groups in percentage of elastin staining at 24 hours (12.9 $\pm 5.5 \%$ vs. $29.1 \pm 21.0 \%$; $14 \mathrm{p}=0.185)$ or 96 hours $(34.0 \pm 22.8$ vs. $14.4 \pm 4.4 \% ; \mathrm{p}=0.143)$, and percentage of 15 collagen staining $(75.6 \pm 11.5 \%$ vs. $70.1 \pm 17 \% \% ; \mathrm{p}=0.611)$ and $(70.5 \pm 15.0 \%$ vs. 75.0 $16 \pm 10.9 \% ; \mathrm{p}=0.638$ ). Representative images are shown in Figure 4.

17

$1911.6 \pm 5.0 \% ; \mathrm{p}=0.612)$ or 96 hours $(16.5 \pm 11.9 \%$ vs. $9.7 \pm 4.7 \% ; \mathrm{p}=0.407)$ and similarly

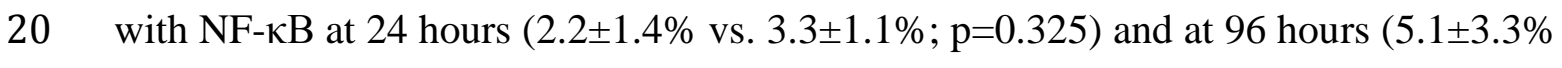
21 vs. $6.9 \pm 3.9 \%$; $=0.559)$. Representative images are shown in Figure 5. 


\section{DISCUSSION}

3 In this study, we set out to establish the nature of bone response to critical illness, and

4 the related functional consequences. In the CLP-exposed rodents there was evidence

5 of early functional changes, compared to sham surgery controls, with a lower

6 maximum load required to fracture the femoral neck at 24 hours. Multi-frequency

7 SPM demonstrated a rapid decrease in collagen elastic modulus at 24 hours, which

8 partially recovered at 96 hours. Mineral elastic modulus was preserved at 24 hours,

9 but decreased significantly at 96 hours.

11 Bone architecture and BMD remained unchanged, as determined by micro-CT.

12 Similarly, histological analysis revealed no differences in bone structure or collagen

13 and elastin content. The observed decrease in bone strength was not accompanied by

14 any change in CXCR4 or NFא $\beta$ expression, or disruption in epiphyseal growth plate

15 organization, osteoblast or osteoclast morphology or quantity and TRAP activity. The

16 CXCR4 pathway plays a crucial role in the osteogenic differentiation of mesenchymal

17 progenitors, and a disruption in its expression or function results in bone epiphyseal

18 growth plate disorganization and abnormal steoblasts development (23). The

19 transcription factor $\mathrm{NF} \kappa \beta$ is a crucial mediator of inflammatory responses, and has

20 been implicated in promoting differentiation of myeloid cells into osteoclasts to

21 exacerbate bone resorption, and to impair bone formation by disrupting osteoblast

22 formation and function (24).

24 The observation of early functional changes to bone strength, in the absence of 25 macroscopic or microscopic changes, reflect the complex organization of bone 
1 structure and the many factors contributing to bone strength. Alterations in matrix

2 composition leading to loss of elastic modulus may effect bone strength, without

3 changing BMD (25), but were excluded by histological analysis. Similarly, the lack

4 of significant pertubations in osteoblast and osteoclast quantity and epiphyseal growth

5 plate thickness and cellular organization, does not support increased bone turnover as

6 an explanation for the early reduction in bone strength we observed.

8 We used multi-frequency scanning probe microscopy (SPM) to analyze changes in

9 bone tissue properties. This technique has been used to study the nano-mechanical 10 properties of a range of biological tissues (26-28) including those of healthy and 11 osteogenesis imperfecta bone $(28,29)$. In the absence of alterations in whole bone 12 morphology, microarchitecture or histology, this nano-scale alteration in bone tissue 13 properties may be an important determinant of the loss of bone mechanical strength 14 (14, 30). Loss of mineral elastic modulus (and corresponding loss of stiffness) may 15 account for the decrease in compression strength over time and loss of collagen elastic 16 modulus (and loss of flexibility and tensile strength) for the reduction in bending 17 strength.

19 The tissue properties of bone that determine bone strength include nature of the 20 collagen, degree and type of collagen cross-linking, size and structure of 21 hydroxyapatite crystals and degree of mineralization. The mineral component of bone 22 is responsible for deformation resistance (31). In critically ill patients, circulating 23 serum calcium has been observed to be associated with loss of bone mineral density 24 acutely (5) and to normalize with recovery (4). This likely represents hydroxyapatite 25 mobilization from mineral stores to maintain normocalcaemia (32). Loss of 
1 hydroxyapatite would therefore lead to loss of mineral elastic modulus, as seen in this

2 rodent model of sepsis. Importantly, crystallinity modulation occurs independently of

3 bone tissue turnover (and NFK $\beta$ signaling) (30).

4

5 The properties of collagen fibers in bone tissue determine energy absorption, an

6 important component of fracture resistance (33). A recent summary of bone turnover

7 marker studies in critical illness (34) demonstrated the consistent increase in urinary

8 markers of loss of collagen mature cross-links - pyridinoline, deoxypyridinoline and

9 collagen type $1 \mathrm{~N}$-Telopeptide (4, 35-39). Loss of collagen cross-link formation is

10 associated with increased fracture risk in non-critical illness pathologies $(40,41)$.

11 Alterations in cross-link formation would not be visible on histological analysis,

12 explaining the lack of changes seen. The end result of loss of these essential

13 intermolecular and interfibrillar cross-links, with likely associated altered collagen

14 fibre orientation (40), would be both a weakening of the extracellular matrix, leading

15 to reduced bending strength (42), and reduced elastic modulus, despite unchanged

16 bone mass (43).

17

18 Trabecular bone has a greater sensitivity to both processes than cortical bone $(44,45)$,

19 leading to functional differences seen between the femoral neck and shaft in our

20 study. Fractures of the femoral head constitute a major personal and public health

21 issue and the loss of bone strength seen offers further biological plausibility to the

22 observed acute and long-term increase in fracture risk described in survivors of

23 critical illness (3-5). 


\section{Clinical Implications}

2 These data answer questions raised following Orford's seminal description of bone

3 loss in critical illness and its population level implications $(3,4)$. The rodents were not

4 exposed to glucocorticoids or sedation, yet both mineral and elastic modulus

5 decreased leading to a significant decrease in force needed to fracture.

6

7 The mechanism of increased bone fragility seems then to be the result of altered

8 biochemical properties of bone, as opposed to bone turnover driven loss of

9 mineralization. Thus, modulation of osteoclastogenesis e.g.(inhibition of Receptor

10 Activator for Nuclear Factor Kappa Beta) or osteoblastogeneisis (e.g. activation of

11 Transforming Growth Factor Beta) seems less likely to be effective mitigation 12 strategies. However, our data suggests that either bispohosphonate therapy (46) or 13 calcium normalization (47), to minimize hydroxyapatite mobilization, may be 14 appropriate interventions. In addition, early mobilization and resistance exercise is 15 likely to be of benefit in the clinical setting, increasing bone strength via alterations in 16 biomechanical properties (48), specifically collagen network organization and 17 deformation resistance, as opposed to increasing bone mineral density (49). Lastly, for 18 critical care survivors, smoking cessation therapy may have a specific role in bone 19 health (50).

\section{$21 \quad$ Limitations}

22 Our study does have important limitations to consider. Extrapolation of rodent data to

23 humans cannot always be done with confidence. However, biological studies on bone 24 metabolism are challenging in humans, more so in the critical care setting. We thus

25 limited our research question to that of the fundamental bone biological response to a 
1 septic insult. The CLP model used had a $0 \%$ rate of mortality, unlike the 55\%

2 reported in $50 \%$ caecal ligation studies (17). Therefore, this model is more likely to

3 represent a mild/moderate form of critical illness. Models with higher mortality and

4 end-organ damage (perhaps more representative of the higher acuity spectrum of

5 critical illness) may demonstrate greater loss of elastic modulus and bone strength.

6 Our animals were sacrificed at 96 hours, and it is possible insufficient time elapsed to

7 accumulate changes in BMD or microarchitecture. However, rodent metabolic and

8 muscle changes can be detected within this time frame (51), and our hypothesis is

9 focused on identifying bone changes in early critical illness, where interventions

10 could modulate bone health in survivors. We did not measure circulating collagen

11 cross-link markers in the model, as this had been well described in humans, and our

12 focus was on altered biomechanical properties and mechanisms of such alterations.

13 Neither did we measure markers of advanced glycation end products which may

14 represent either Acute Lung Injury (52) or increased post-transcriptional modification

15 of new collagen fibres $(44,53,54)$. Future directions for animal model work might

16 include exploration of therapies to modulate inorganic matrix mobilization and loss of

17 collagen cross-links.

19 Despite the lower acuity and limited time frame, differences in elastic modulus and

20 bone strength were demonstrated. Whilst no formal power calculation was performed,

21 AFM scanning microscopy comparing bone stiffness in wild-type mice to those with

22 osteogenesis imperfecta, has detected significant differences using a sample size of 2

23 (28). We expected a larger standard deviation in our CLP group, therefore a larger

24 sample size was selected, consistent with other animal publications in critical illness

25 (55-57). Despite this, in humans, patchy myonecrosis has been seen affecting different 
1 areas of muscle (58)- if the same were true for bone, histological and immunostaining

2 data reported in this manuscript may be at risk of a Type II error. Our data suggest the

3 need for larger studies on animal models (induced with a higher acuity of critical

4 illness) or human subjects where sustainable interventions can be additionally be 5 assessed.

6

7 In conclusion, femoral neck strength is reduced in a rodent model of sepsis, with 8 associated decreases in both collagen and mineral modulus. The mechanism of this 9 phenomena is likely to be altered biomechanical properties instead of increased bone 10 turnover.

\section{ACKNOWLEDGEMENTS}

13 We acknowledge help and support with reading the histology slides from Dr Victor 14 Lee MD PhD, Department of Pathology at National University Singapore.

16 Figure 1

17 Femur mechanical strength at neck (A) and shaft of femur (B). Compressive and 18 bending strength at the neck of femur is also shown ( $\mathrm{C}$ and $\mathrm{D}$, respectively). *Denotes 19 data is significantly different from sham control subjects $(\mathrm{p}<0.05)$. $†$ Cecal ligation 20 and puncture. $\mathrm{N}=\mathrm{Newtons}$

\section{Figure 2}

23 (A) Collagen elastic modulus for sham, CLP(24) and CLP(96) groups. (B) Mineral 24 elastic modulus for sham, CLP(24) and CLP(96) groups. *Denotes data is 
1 significantly different from sham control subjects $(\mathrm{p}<0.05)$. $†$ Cecal ligation and

2 puncture.

3

$4 \quad$ Figure 3

5 Representative images from multi-frequency scanning probe microscopy (SPM) and

6 microCT reconstruction. Multi-frequency SPM was used to image collagen (A) and

7 mineral (B) elastic modulus. MicroCT images were analysed in coronal (C) and

8 transverse (D) views.

9

$10 \quad$ Figure 4

11 Representative histology images showing results of hemotoxylin and eosin (H\&E)

12 staining, as well as staining for collagen (Masson's trichrome stain) and elastin 13 (Verhoeff stain). Scale bar is $100 \mu \mathrm{M}$.

$15 \quad$ Figure 5

16 Immunohistochemistry images using antibody staining for Chemokine Receptor type 174 (CXCR4), Nuclear Factor Kappa Beta (NF-kB)and Tartarate Resistant Acid 18 Phosphatase (TRAP) at the femoral neck. Scale bar is $100 \mu \mathrm{M}$.

19

20 References

21

22 1. Elliott D, Davidson JE, Harvey MA, et al.: Exploring the scope of post23 intensive care syndrome therapy and care: engagement of non-critical care 24 providers and survivors in a second stakeholders meeting. Critical Care $25 \quad$ Medicine 2014; 42:2518-2526 
12 2. Griffith DM, Walsh TS: Bone loss during critical illness: a skeleton in the

$2 \quad$ closet for the intensive care unit survivor? Critical Care Medicine 2011;

$3 \quad 39: 1554-1556$

43 . Orford NR, Saunders K, Merriman E, et al.: Skeletal morbidity among $5 \quad$ survivors of critical illness. Critical Care Medicine 2011; 39:1295-1300

64 Orford NR, Lane SE, Bailey M, et al.: Changes in Bone Mineral Density in the $7 \quad$ Year after Critical Illness. Am J Respir Crit Care Med 2016; 193:736-744

8 5. Rawal J, McPhail MJW, Ratnayake G, et al.: A pilot study of change in 9 fracture risk in patients with acute respiratory distress syndrome. Crit Care $10 \quad 2015 ; 19: 165$

11 6. Bilezikian JP: Bone Loss in the Intensive Care Unit. Am J Respir Crit Care $12 \quad$ Med 2016; 193:706-707

13 7. Fletcher SN, Kennedy DD, Ghosh IR, et al.: Persistent neuromuscular and 14 neurophysiologic abnormalities in long-term survivors of prolonged critical $15 \quad$ illness. Critical Care Medicine 2003; 31:1012-1016

16 8. Burtin C, Clerckx B, Robbeets C, et al.: Early exercise in critically ill patients 17 enhances short-term functional recovery. Critical Care Medicine 2009; $18 \quad 37: 2499-2505$

19 9. Kress JP, Hall JB: ICU-acquired weakness and recovery from critical illness. N $20 \quad$ Engl J Med 2014; 370:1626-1635

21 10. Puthucheary ZA, Rawal J, McPhail M, et al.: Acute skeletal muscle wasting in $22 \quad$ critical illness. JAMA 2013; 310:1591-1600

23 11. Chang J, Wang Z, Tang E, et al.: Inhibition of osteoblastic bone formation by 24 nuclear factor-kappaB. Nat Med 2009; 15:682-689

25 12. Yellowley C: CXCL12/CXCR4 signaling and other recruitment and homing 
$1 \quad$ pathways in fracture repair. Bonekey Rep 2013; 2:300

2 13. Keaveny TM, Kopperdahl DL, Melton LJ, et al.: Age-dependence of femoral strength in white women and men. J Bone Miner Res 2010; 25:994-1001

4 14. Fonseca H, Moreira-Gonçalves D, Coriolano H-JA, et al.: Bone Quality: The Determinants of Bone Strength and Fragility. Sports Med 2013; 44:37-53

15. Elkin SL, Vedi S, Bord S, et al.: Histomorphometric analysis of bone biopsies 2002; 166:1470-1474

16. Vincent J-L, Sakr Y, Sprung CL, et al.: Sepsis in European intensive care units: Results of the SOAP study*. Critical Care Medicine 2006; 34:344-353

17. Rittirsch D, Huber-Lang MS, Flierl MA, et al.: Immunodesign of experimental sepsis by cecal ligation and puncture. Nat Protoc 2009; 4:31-36

18. Garcia R, Herruzo ET: The emergence of multifrequency force microscopy. Nature Nanotechnology 2012; 7:217-226

19. Wallace JM: Applications of atomic force microscopy for the assessment of nanoscale morphological and mechanical properties of bone. Bone 2012; $50: 420-427$

20. Stark RW: Dynamics of repulsive dual-frequency atomic force microscopy. Applied Physics Letters 2009; 94:063109

21. Doube M, Kłosowski MM, Arganda-Carreras I, et al.: BoneJ: Free and extensible bone image analysis in ImageJ. Bone 2010; 47:1076-1079

22. Schneider CA, Rasband WS, Eliceiri KW: NIH Image to ImageJ: 25 years of image analysis. Nat Methods 2012; 9:671-675

23. Zhu W, Liang G, Huang Z, et al.: Conditional inactivation of the CXCR4 receptor in osteoprecursors reduces postnatal bone formation due to impaired 
$1 \quad$ osteoblast development. J Biol Chem 2011; 286:26794-26805

2 24. Swarnkar G, Zhang K, Mbalaviele G, et al.: Constitutive activation of IKK2/NF- $\kappa$ B impairs osteogenesis and skeletal development. PLoS ONE 2014;

4 9:e91421

5 25. Burr DB, Robling AG, Turner CH: Effects of biomechanical stress on bones in animals. Bone 2002; 30:781-786

7 26. Hansma HG, Kim KJ, Laney DE, et al.: Properties of biomolecules measured

27. Hilal N, Bowen WR, Alkhatib L, et al.: A Review of Atomic Force Microscopy Applied to Cell Interactions with Membranes. Chemical Engineering Research and Design 2006; 84:282-292

28. Li T, Chang S-W, Rodriguez-Florez N, et al.: Studies of chain substitution caused sub-fibril level differences in stiffness and ultrastructure of wildtype and oim/oim collagen fibers using multifrequency-AFM and molecular modeling. Biomaterials 2016; 107:15-22

29. User $\mathrm{N}$, Thompson $\mathrm{JB}$, Kindt $\mathrm{JH}$, et al.: Bone indentation recovery time correlates with bond reforming time. Nature 2001; 414:773-776

30. Boskey AL, Coleman R: Aging and bone. J Dent Res 2010; 89:1333-1348

31. Waring R, Seeman E, Delmas PD: Bone quality--the material and structural basis of bone strength and fragility. N Engl J Med 2006; 354:2250-2261

32. Lieben L, Masuyama R, Torrekens S, et al.: Normocalcemia is maintained in mice under conditions of calcium malabsorption by vitamin $\mathrm{D}$-induced inhibition of bone mineralization. J Clin Invest 2012; 122:1803-1815 
$1 \quad$ properties of collagen. J Struct Biol 1998; 122:119-122

2 34. Lee P, Nair P, Eisman JA, et al.: Bone Failure in Critical Illness. Critical Care

3 Medicine 2016; 44:2270-2274

35. Smith LM, Cuthbertson B, Harvie J, et al.: Increased bone resorption in the critically ill: association with sepsis and increased nitric oxide production. Critical Care Medicine 2002; 30:837-840

36. Van den Berghe G, Baxter RC, Weekers F, et al.: The combined administration of GH-releasing peptide-2 (GHRP-2), TRH and GnRH to men with prolonged critical illness evokes superior endocrine and metabolic effects compared to treatment with GHRP-2 alone. Clin Endocrinol (Oxf) 2002; 56:655-669

37. Van den Berghe G, Van Roosbroeck D, Vanhove P, et al.: Bone turnover in prolonged critical illness: effect of vitamin D. J Clin Endocrinol Metab 2003; $88: 4623-4632$

38. Nierman DM, Mechanick JI: Bone hyperresorption is prevalent in chronically critically ill patients. Chest 1998; 114:1122-1128

39. Nierman DM, Mechanick JI: Biochemical response to treatment of bone hyperresorption in chronically critically ill patients. Chest 2000; 118:761-766

40. Martin E, Shapiro JR: Osteogenesis imperfecta: Epidemiology and pathophysiology. Current Osteoporosis Reports 2009; 5:91-97

41. Simon JA, Hudes ES: Relation of ascorbic acid to bone mineral density and self-reported fractures among US adults. Am J Epidemiol 2001; 154:427-433

42. Bailey AJ, Sims TJ, Ebbesen EN, et al.: Age-related changes in the biochemical properties of human cancellous bone collagen: relationship to bone strength. Calcified Tissue International 1999; 65:203-210 
1 cancellous bone: correlation with collagen intermolecular cross-links. J Bone Miner Res 2002; 17:1621-1628

3 44. Viguet-Carrin S, Follet H, Gineyts E, et al.: Association between collagen

47. Worth H, Stammen D, Keck E: Therapy of steroid-induced bone loss in adult asthmatics with calcium, vitamin D, and a diphosphonate. Am J Respir Crit Care Med 1994; 150:394-397

48. Huang T-H, Chang F-L, Lin S-C, et al.: Endurance treadmill running training benefits the biomaterial quality of bone in growing male Wistar rats. J Bone Miner Metab 2008; 26:350-357

49. Shiiba M, Arnaud SB, Tanzawa H, et al.: Regional alterations of type I collagen in rat tibia induced by skeletal unloading. J Bone Miner Res 2002; $17: 1639-1645$

50. Terashima T, Wiggs B, English D, et al.: The effect of cigarette smoking on the bone marrow. Am J Respir Crit Care Med 1997; 155:1021-1026

51. O'Leary MJ, Ferguson CN, Rennie MJ, et al.: Sequential changes in in vivo muscle and liver protein synthesis and plasma and tissue glutamine levels in sepsis in the rat. Clin Sci 2001; 101:295-304 
1 52. Calfee CS, Ware LB, Eisner MD, et al.: Plasma receptor for advanced glycation end products and clinical outcomes in acute lung injury. Thorax 2008; 63:1083-1089

4 53. Saito M, Marumo K: Collagen cross-links as a determinant of bone quality: a possible explanation for bone fragility in aging, osteoporosis, and diabetes mellitus. Osteoporos Int 2010; 21:195-214

7 54. Santana RB, $\mathrm{Xu} \mathrm{L}$, Chase $\mathrm{HB}$, et al.: A role for advanced glycation end 8 products in diminished bone healing in type 1 diabetes. Diabetes 2003;

10 55. Patel BV, Wilson MR, O'Dea KP, et al.: TNF-induced death signaling triggers 11 alveolar epithelial dysfunction in acute lung injury. J Immunol 2013;

13 56. Files DC, D'Alessio FR, Johnston LF, et al.: A critical role for muscle ring finger-1 in acute lung injury-associated skeletal muscle wasting. Am J Respir 
A

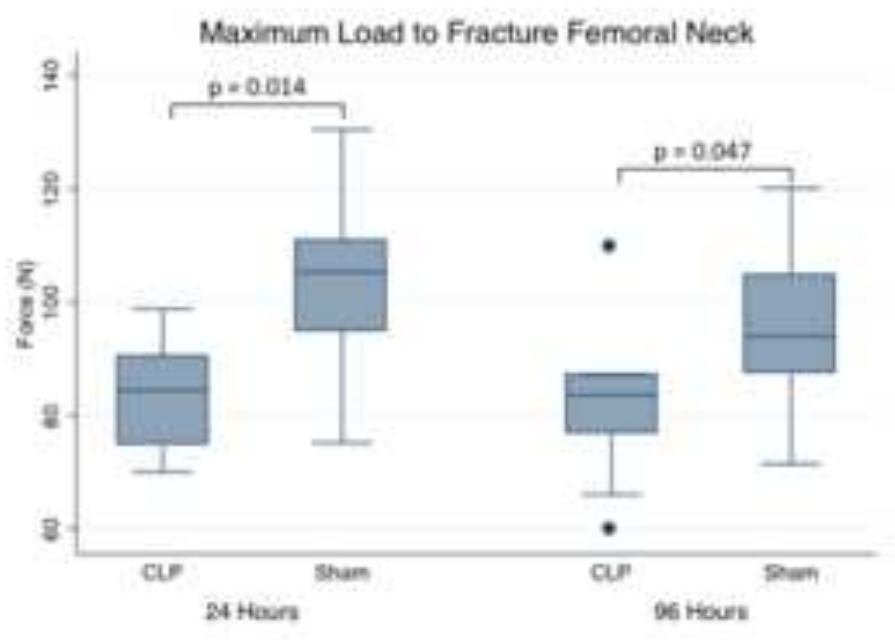

C

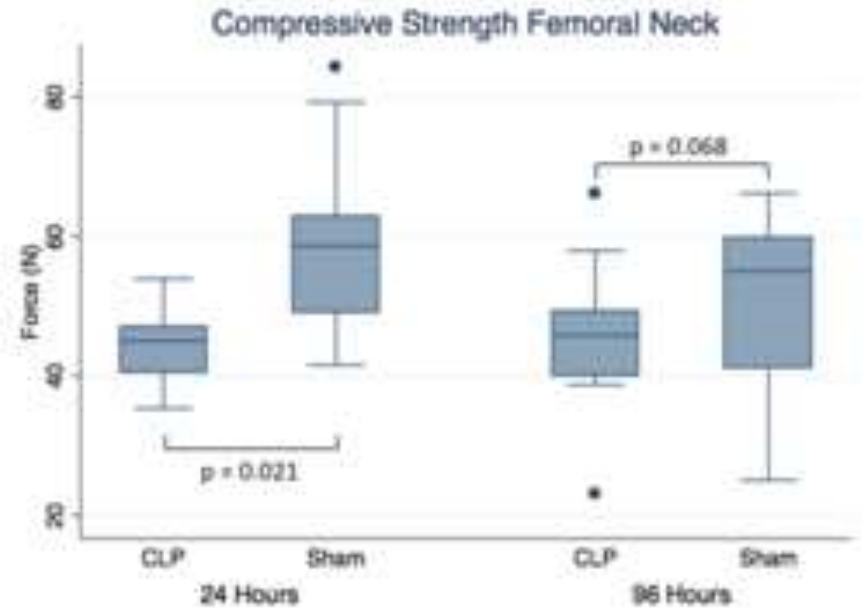

B

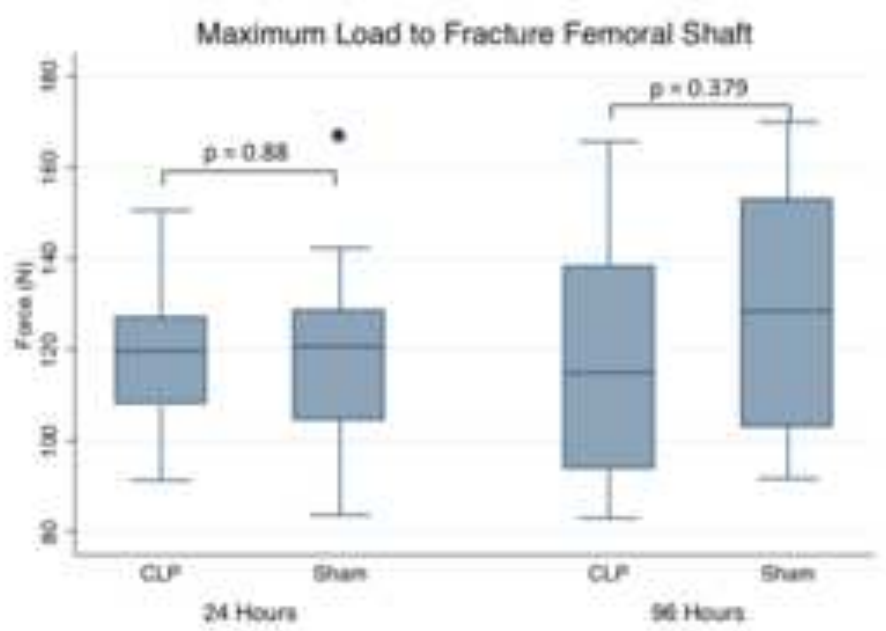

D

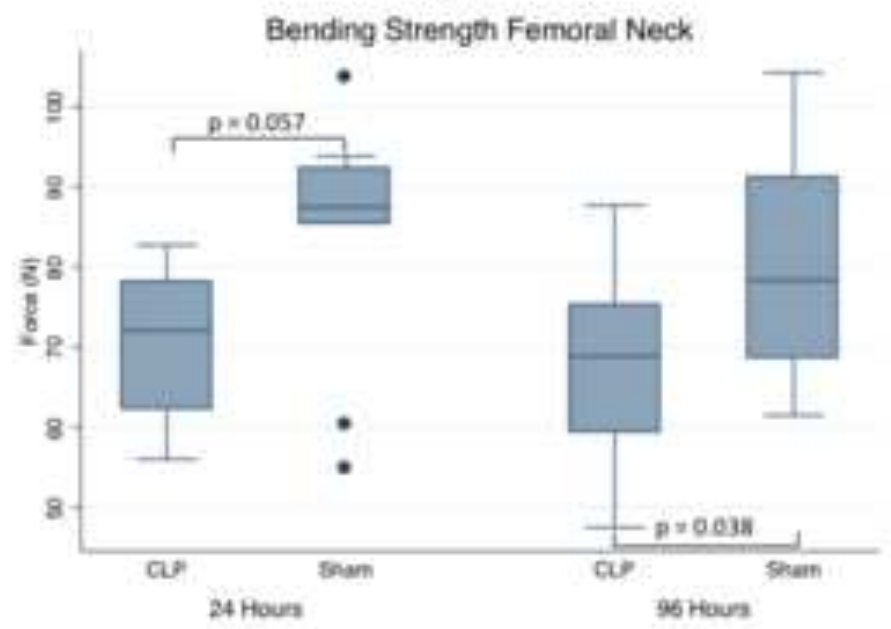


A

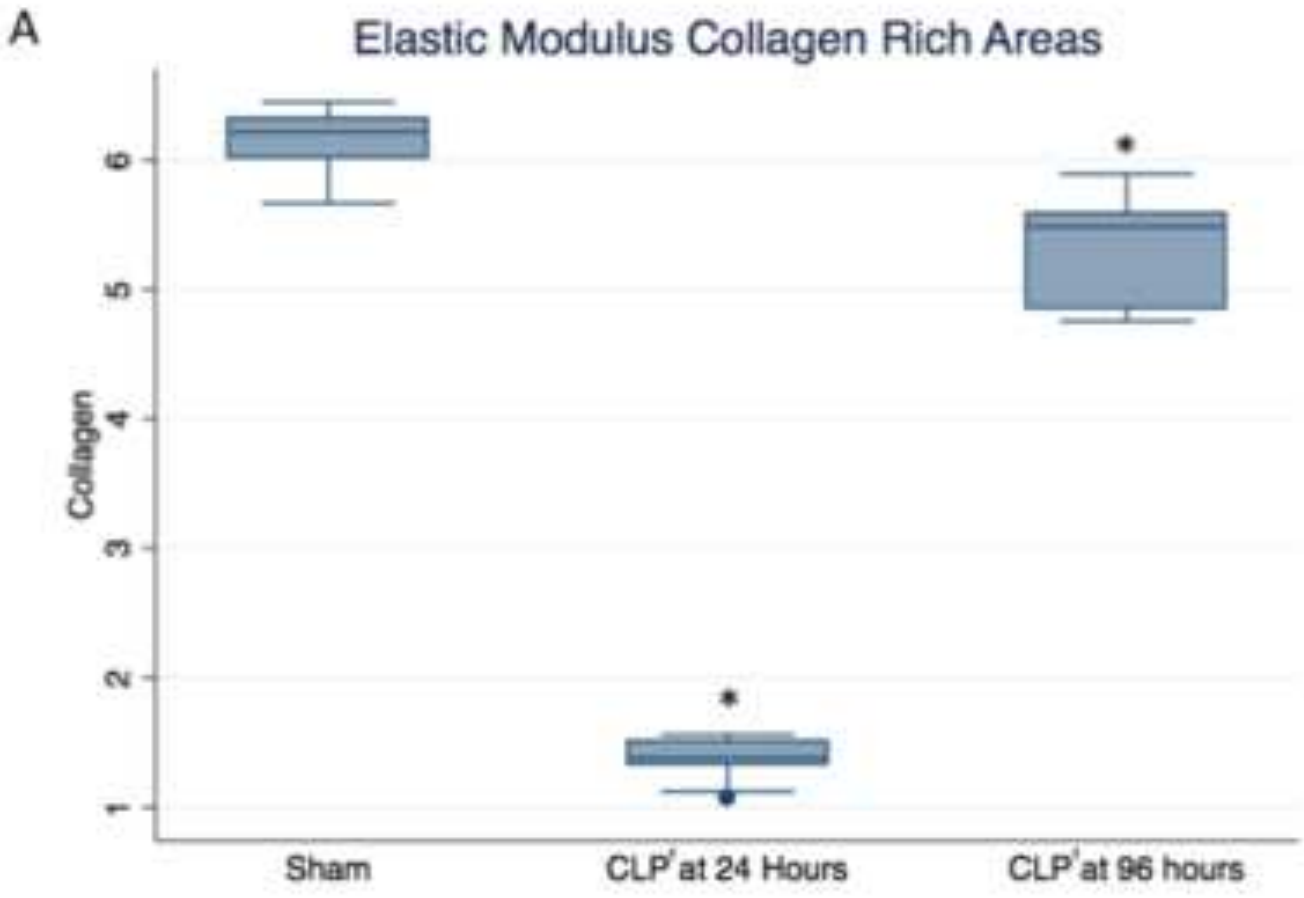

B

Elastic Modulus Mineral Rich Areas

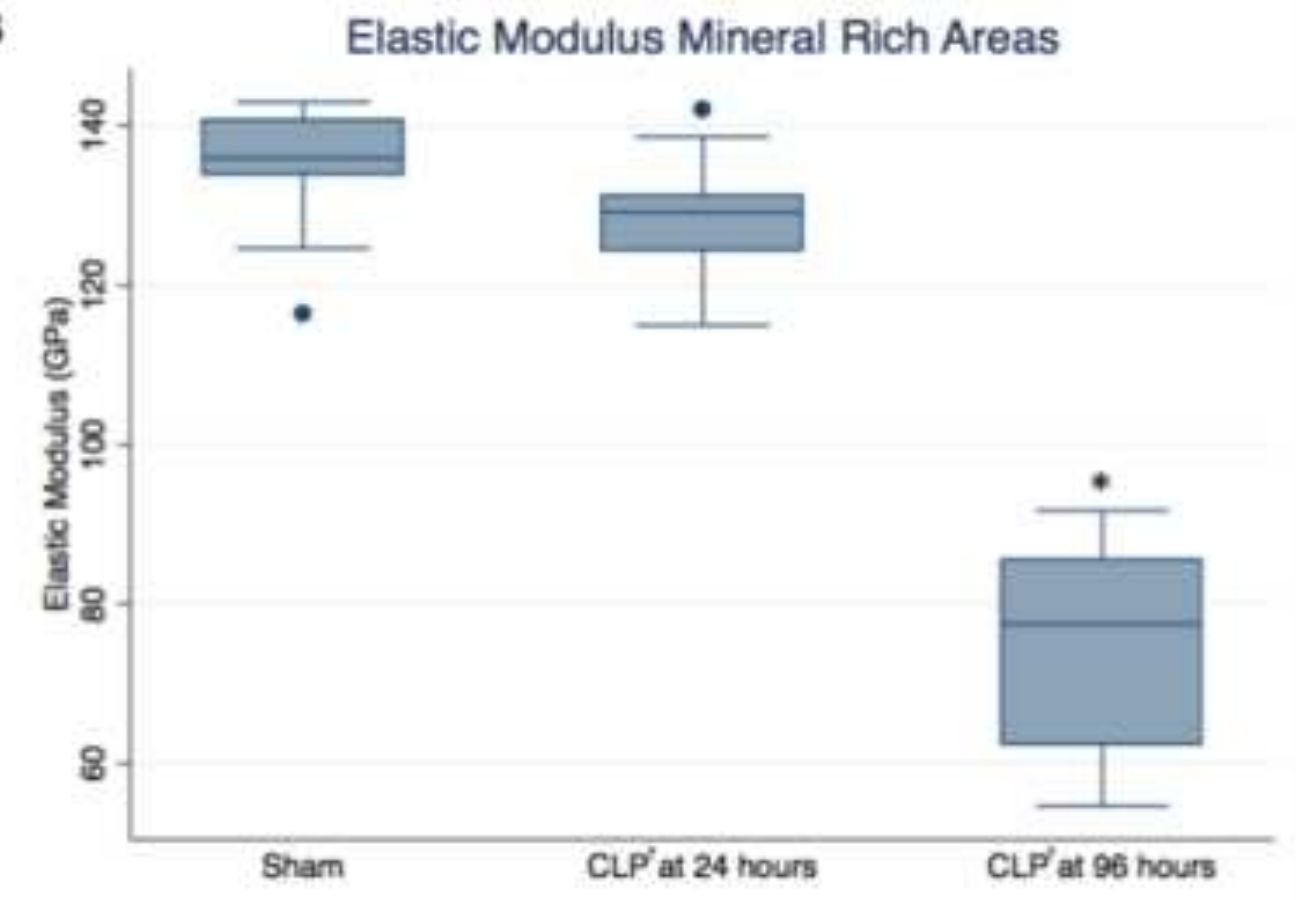




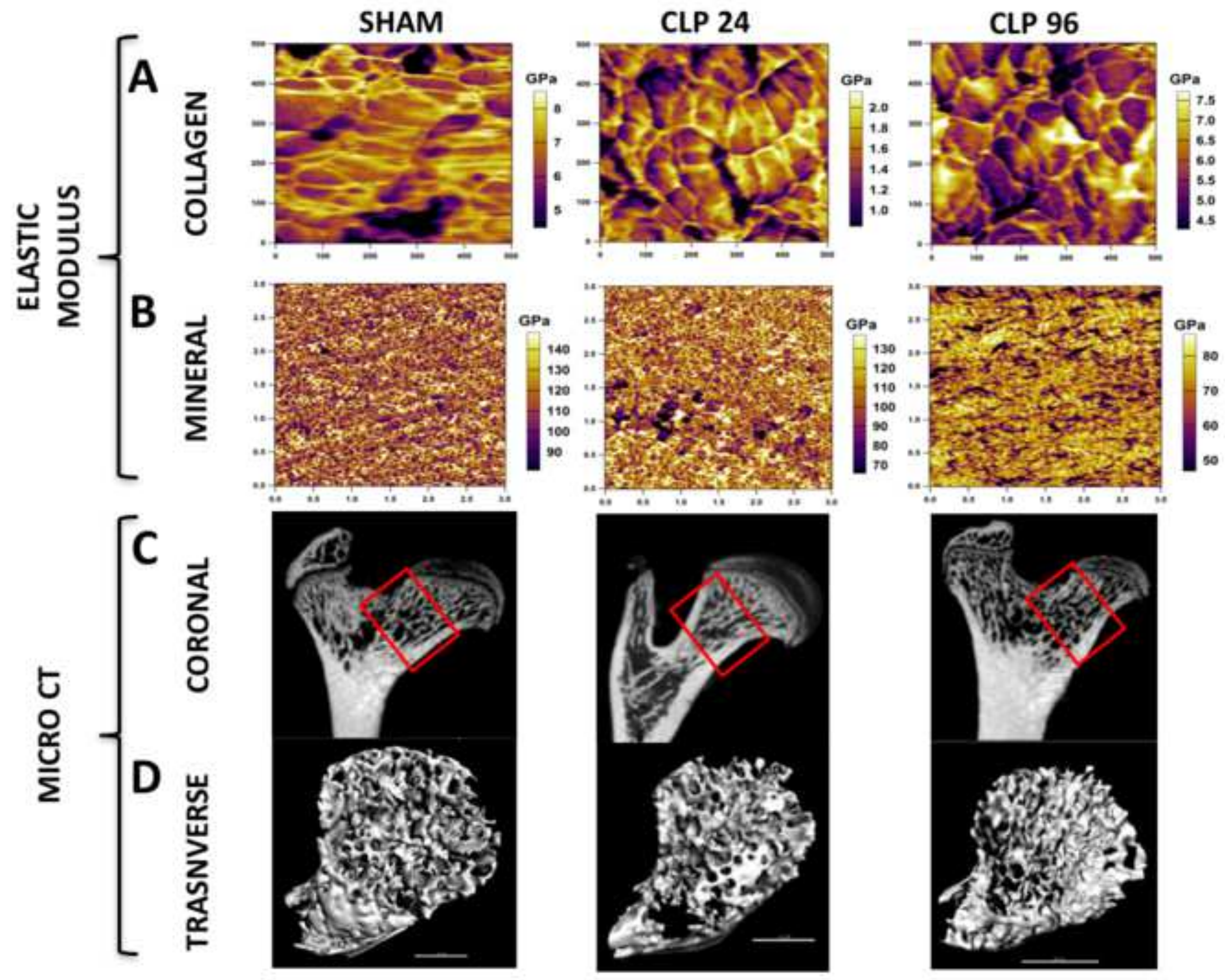




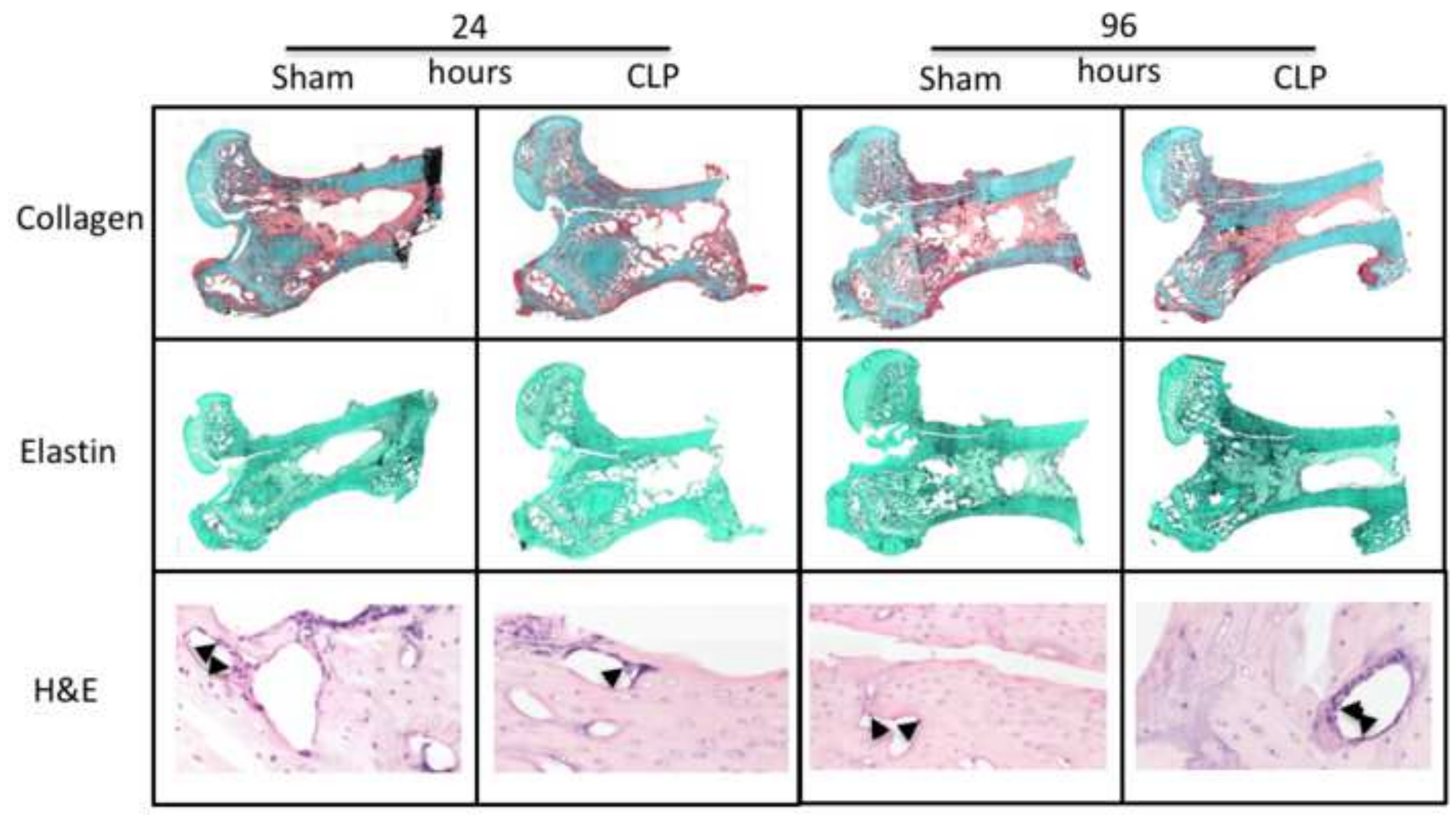




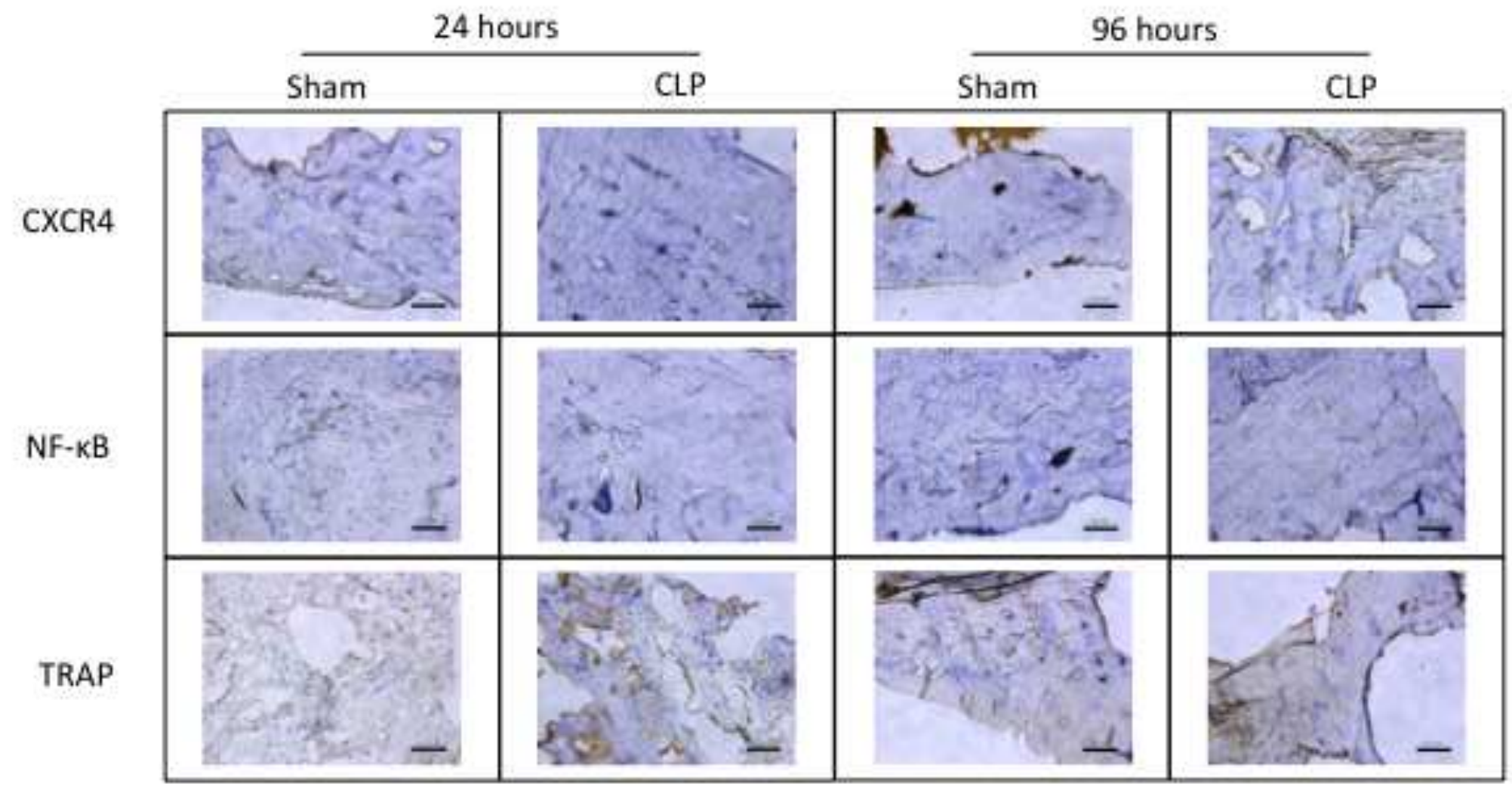


Online supplement
\[ \text { Supplemental Data File (.doc, .tif, pdf, etc.) } \]
Supplementrodent boneCCM.docx

Online supplement
\[ \text { Supplemental Data File (.doc, .tif, pdf, etc.) } \]
Supplementrodent boneCCM.docx Online supplement
\[ \text { Supplemental Data File (.doc, .tif, pdf, etc.) } \]
Supplementrodent boneCCM.docx

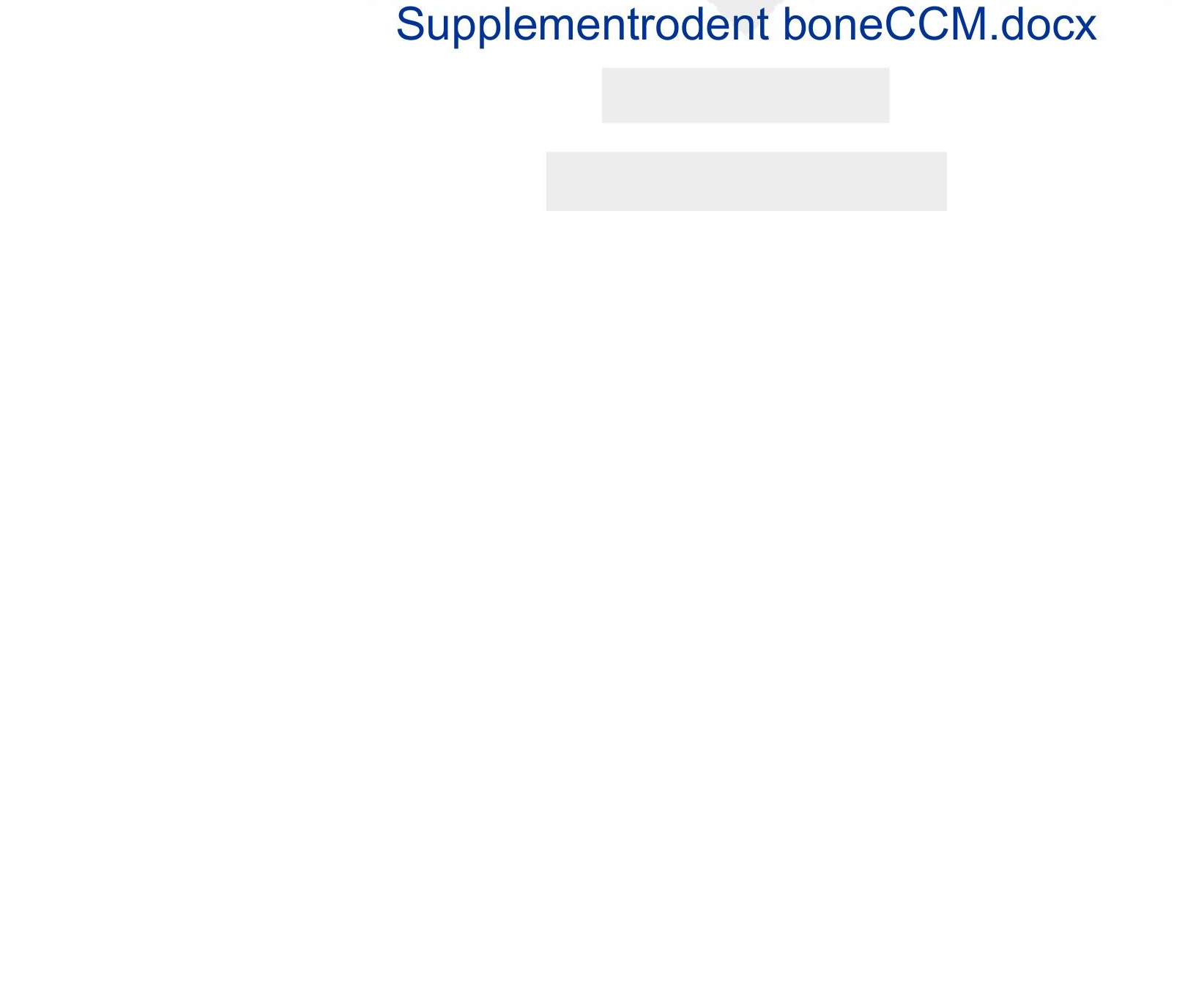

.

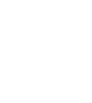

